\title{
Full-pose Manipulation Control of a Cable-suspended load with Multiple UAVs under Uncertainties
}

\author{
D. Sanalitro ${ }^{1}$, H. J. Savino ${ }^{1}$, M. Tognon ${ }^{1}$, J. Cortés ${ }^{1}$, A. Franchi ${ }^{2,1}$
}

\begin{abstract}
In this work we propose an uncertainty-aware controller for the Fly-Crane system, a statically rigid cablesuspended aerial manipulator using the minimum number of aerial robots and cables. The force closure property of the FlyCrane makes it ideal for applications where high precision is required and external disturbances should be compensated. The proposed control requires the knowledge of the nominal values of a minimum number of uncertain kinematic parameters, thus simplifying the identification process and the controller implementation. We propose an optimization-based tuning method of the control gains that ensures stability despite parameter uncertainty and maximizes the $H_{\infty}$ performance. The validity of the proposed framework is shown through real experiments.
\end{abstract}

\section{INTRODUCTION}

Unmanned Aerial Vehicles (UAVs) are nowadays used in different domains, from search \& rescue and inspection to precise agriculture and transportation of loads [1]. In the latter case, the object is either rigidly attached to the vehicle [2] or attached using a cable [3]. Multiple aerial vehicles rigidly attached to the load [4] or attached to the load's center of mass (CoM) using cables [5] are typically used to overcome limited payload of the single-vehicle solution.

In the last few years, motivated by manipulation tasks such as assembly, construction, contact-based inspection, etc., the focus moved toward the more complex problem of controlling the load full pose, i.e., both position and orientation. In most of the manipulation tasks, the full pose control is a strong requirement, e.g., transporting objects that cannot enter in limited spaces unless tilting them, or for pegin-hole operations where just controlling the position of the load is not enough.

Among several solutions, the one based on cables is the most suitable for the mentioned applications thanks to the induced decoupling between the rotational dynamics of the vehicles and the one of the load. Full pose control of the load is possible when the robotic system can attain a sixdimensional wrench on the load by controlling the orientation of the cables and the force applied by them (called stress). The minimum setup that possesses such ability is a system with three vehicles connected to three non-collinear points

\footnotetext{
${ }^{1}$ LAAS-CNRS, Université de Toulouse, CNRS, Toulouse, France, dario.sanalitro@laas.fr, heitor.judiss-savino@laas.fr, marco.tognon@laas.fr, juan. cortes@laas. fr.

${ }^{2}$ Robotics and Mechatronics lab, Faculty of Electrical Engineering, Mathematics \& Computer Science, University of Twente, Enschede, The Netherlands a.franchi@utwente.nl

This research was partially supported by the ANR, Project ANR17-CE33-0007 MuRoPhen, and by the European Union's Horizon 2020 research and innovation programme under grant agreement ID: 871479 AERIAL-CORE
}

on the load through three cables [6] (see Fig. 1 (a) for a schematic example, and [7] for additional details). The main drawback of this setup is that the compensation of a time-varying external disturbing wrench (e.g., wind) must be obtained by moving the three aerial vehicles to reorient the three cables. This makes the overall system sluggish and imprecise in real-world conditions. To overcome such drawback one has to employ a statically rigid [8] (or forceclosure [6]) design. Such design allows compensating timevarying external wrenches almost instantaneously by varying only the stresses while keeping all the cables in the same orientation. Six is the minimum number of cables in order to gain such a property. A possible setup is the one using six cable-robot pairs, as in Fig. 1 (b).

However, the use of six aerial vehicles considerably increases the overall system complexity and costs compared to the three aerial vehicle case. A simpler and more efficient choice consists in using only three aerial vehicles connected to a pair of cables each, see Fig. 1 (c). This statically rigid system is called the Fly-Crane and was first presented in [9].

Several algorithms have been proposed to control the pose of a cable-suspended load handled by multiple UAVs. In [10], [11] and [12], position and yaw angle of a slung payload are controlled in real scenarios. In all these works, as in [13], regulation is achieved using a communicationbased formation control of the UAVs. A communicationless approach, which relies on the sensing of contact forces, is instead proposed in [14]. Differently, in [7] the UAVs follow in open loop the nominal trajectories computed from the desired load trajectory using differential flatness. In order to cope with the high sensitivity to model errors and external disturbances of open-loop approaches, other works employ geometric control techniques [15] or feedback control laws that require the inversion of the Full Dynamic Model (FDM) [16]. Although the feedback on the load's pose makes the overall approach more robust to non-ideal scenarios, these methods still require a good knowledge of all the dynamic model parameters. However, it is known that if feedback controlled systems do not take model uncertainties into account, stability may be lost in real world conditions. Few works started to address this problem, e.g., [10] showed the robustness of the system to external disturbances, [15] suppresses undesired behaviors when facing random uncertainties, [12] and [17] consider the payload itself as a disturbance to be controlled.

Departing from the current state of the art, the main contribution of this work is to propose a novel algorithm aiming at the full-pose control of the load for the Fly- 


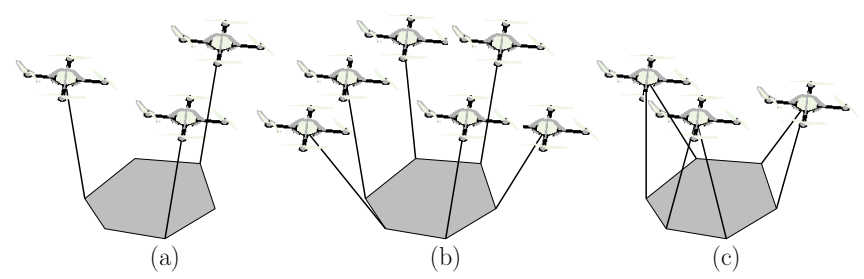

Fig. 1: Cable-suspended load configurations: (a) Three robots and three cables [7]; (b) Six robots and six cables [16]; (c) Three robots and six cables (the Fly-Crane).

Crane cable-suspended aerial manipulator. The proposed method does not rely on the dynamical model and formally takes into account model parameter uncertainty and external disturbances in its design phase. The advantage of reasoning at the kinematic level rather than at the dynamic one is that we need to identify a smaller set of parameters. Furthermore, the parameters are related only to geometric quantities, which are easier to measure with high accuracy. Furthermore, in our approach, the dynamics of the system is not completely neglected, but all its effects are rather considered as disturbances, and formally addressed in the design phase of the controller. As a drawback, to limit disturbances, and so tracking errors, the acceleration of the desired platform motion should be kept low. On the contrary, an FDM controller theoretically allows acrobatic motions as well. Having quasi-static trajectories is the price to pay to obtain a simpler and more precise identification process of the system parameters. This is not a real problem for the considered manipulation-related applications (e.g., construction and assembly), where accuracy is preferred over agility.

Relying on an inner/outer loop approach, we designed the proposed controller such that it is simple to implement in real world. In the inner loop, the three UAVs aim at regulating their velocities using simple decentralized proportional controllers. The outer loop provides velocity references to the inner loop in order to regulate the system configuration, including the full pose of the load. We demonstrate how the control gain of the outer loop can be designed to: i) cope with the parametric uncertainty of the system model and ensure stability despite the non-perfect knowledge of its kinematic parameters; ii) minimize the effect of external disturbances by optimizing the $H_{\infty}$ performance index. The proposed framework is tested by real indoor experiments under different conditions.

The paper is organized as follows. Section II describes the Fly-Crane system and its modeling. The proposed robust controller is presented in Sec. III. The experimental results are shown in Sec. IV and on the attached multimedia material. Final discussions and future works are presented in Sec. V.

\section{THE FLY-CRANE SYSTEM}

For the reasons mentioned above, we believe that the Fly-Crane is one of the most suitable systems for aerial manipulation. The Fly-Crane system is composed by three aerial vehicles and a platform, which is in turn constituted by a load and six cables arranged as in Fig. 2. In order to define the Fly-Crane model upon which the robust controller will be designed, let us define an inertial frame $\mathcal{F}_{W}=\left\{O_{W}, \boldsymbol{x}_{W}, \boldsymbol{y}_{W}, \boldsymbol{z}_{W}\right\}$, where $O_{W}$ is its origin and $\left\{\boldsymbol{x}_{W}, \boldsymbol{y}_{W}, \boldsymbol{z}_{W}\right\}$ are its unit axes. Then we define another frame $\mathcal{F}_{L}=\left\{O_{L}, \boldsymbol{x}_{L}, \boldsymbol{y}_{L}, \boldsymbol{z}_{L}\right\}$ rigidly attached to the load. $\mathcal{F}_{L}$ is placed such that $O_{L}$ is centered on the CoM of the load. The configuration of the load is then given by the vector ${ }^{W} \boldsymbol{p}_{L}=\left[\boldsymbol{p}_{L_{x}}, \boldsymbol{p}_{L_{y}}, \boldsymbol{p}_{L_{z}}\right] \in \mathbb{R}^{3}$, describing the position of $O_{L}$ with respect to (w.r.t.) $\mathcal{F}_{W}$, and by the Euler angles ${ }^{W} \boldsymbol{\eta}_{L}=[\phi, \theta, \psi] \in \mathbb{R}^{3}$ describing the orientation of $\mathcal{F}_{L}$ w.r.t. $\mathcal{F}_{W}{ }^{1}$. We complete the description of the load state with its linear velocity $\boldsymbol{v}_{L}=d \boldsymbol{p}_{L} / d t \in \mathbb{R}^{3}$ w.r.t. $\mathcal{F}_{W}$, and the Euler angles velocities $\dot{\boldsymbol{\eta}}_{L} \in \mathbb{R}^{3}$.

The $i$-th cable is attached at one end to the load at point $B_{i}$ and at the other end to an aerial vehicle at point $O_{R i}$. The position of $B_{i}$ and $O_{R i}$ w.r.t. $\mathcal{F}_{W}$ are described by the vectors $\boldsymbol{b}_{i} \in \mathbb{R}^{3}$ and $\boldsymbol{p}_{R i} \in \mathbb{R}^{3}$, respectively. Since each pair of cables $(1,2),(3,4)$ and $(5,6)$ is attached to the same point of the same aerial vehicle, we have that $O_{R 1} \equiv O_{R 2}, O_{R 3} \equiv$ $O_{R 4}$ and $O_{R 5} \equiv O_{R 6}$. Connections between cable-load and cable-robot are done such that no rotational constraints are present. We assume that all the points $B_{i}$ lie on the plane $\left\{\boldsymbol{x}_{L}, \boldsymbol{y}_{L}\right\}$. As normally done in the state of the art, we assume in the model that the cables have negligible mass and inertia w.r.t. the other bodies of the system. The model also neglects phenomena like sagging and elongation and assumes the cables always taut ${ }^{2}$. Thus the generic $i$-th cable, with $i=1, \ldots, 6$, has a constant length $l_{i} \in \mathbb{R}_{>0}$ in the model. These assumptions are also motivated by the fact that for a Fly-Crane system, the cables are never very long with respect to the dimensions of the system. In fact, differently from cable-driven robots, the Fly-Crane possesses a large positional workspace regardless of the length of the cables. Furthermore, long cables are undesirable because they make the two-cable vehicle-load connection similar to a singlecable connection (see Fig. 1 (a)), at the detriment of the sought static rigidity property.

The configuration of the pair of cables $(i, j) \in$ $\{(1,2),(3,4),(5,6)\}$ with respect to $\mathcal{F}_{L}$ is represented by the angle $\alpha_{i j} \in \mathbb{R}$ between the plane $\left\{\boldsymbol{x}_{L}, \boldsymbol{y}_{L}\right\}$ and the plane formed by the cables $(i, j)$. The platform configuration is then entirely described by the variable $\boldsymbol{q}=\left[\boldsymbol{p}_{L}^{\top} \boldsymbol{\eta}_{L}^{\top} \boldsymbol{\alpha}^{\top}\right]^{\top} \in$ $\mathcal{C} \subset \mathbb{R}^{9}$, where $\boldsymbol{\alpha}=\left[\begin{array}{lll}\alpha_{12} & \alpha_{34} & \alpha_{56}\end{array}\right]^{T} \in \mathbb{R}^{3}$.

The three positions of the robots, $\boldsymbol{p}_{R i} \in \mathbb{R}^{3}$, with $i=$ $1,2,3$, depend on $\boldsymbol{q}$ through the kinematic relation:

$$
\boldsymbol{p}_{R i}(\boldsymbol{q})=\boldsymbol{p}_{L}+\boldsymbol{R}_{L}\left(\boldsymbol{\eta}_{L}\right)^{P} \boldsymbol{p}_{R i}\left(\alpha_{i j}\right)
$$

where $\boldsymbol{R}_{L}\left(\boldsymbol{\eta}_{L}\right) \in S O(3)$ is the rotation matrix computed

\footnotetext{
${ }^{1}$ The left superscript indicates the reference frame. From now on, $\mathcal{F}_{W}$ is considered as reference frame when the superscript is omitted.

${ }^{2}$ All the non-idealities and parametric uncertainties in the model will be dealt with in the robust control design, and validated in real experiments. Furthermore, the tautness is ensured using a motion planner, like the one in [9], that provides configurations of reference that always ensure taut cables.
} 


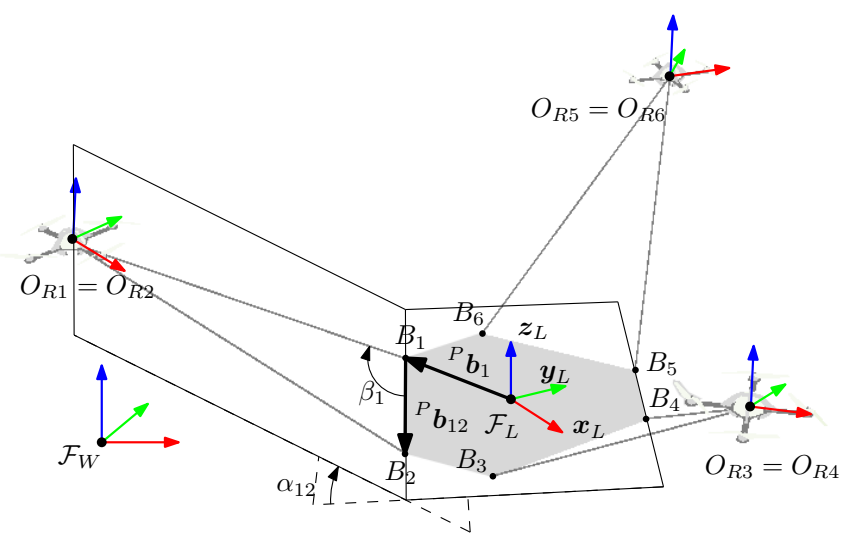

Fig. 2: Schematic representation of the Fly-Crane and its main variables.

from $\boldsymbol{\eta}_{L},{ }^{P} \boldsymbol{p}_{R i}={ }^{P} \boldsymbol{b}_{i}+l_{i} \boldsymbol{R}_{{ }^{P} \boldsymbol{b}_{i j}}\left(\alpha_{i j}\right) \boldsymbol{R}_{\boldsymbol{z}_{L}}\left(\beta_{i}\right) \frac{{ }^{P} \boldsymbol{b}_{i j}}{\left\|{ }^{P} \boldsymbol{b}_{i j}\right\|},{ }^{P} \boldsymbol{b}_{i j}$ is the position of the vector $\overrightarrow{B_{i} B_{j}}$ expressed in $\mathcal{F}_{L}$, and $\beta_{i} \in \mathbb{R}$ is the angle between $\overrightarrow{B_{i} B_{j}}$ and $\overrightarrow{B_{i} O_{R i}}$. From (1) we can compactly define the kinematic model of the system as:

$$
\boldsymbol{p}_{R}=\boldsymbol{f}(\boldsymbol{q}),
$$

where $\boldsymbol{p}_{R}=\left[\begin{array}{lll}\boldsymbol{p}_{R 1}^{\top} & \boldsymbol{p}_{R 3}^{\top} & \boldsymbol{p}_{R 5}^{\top}\end{array}\right]^{\top}$. Differentiating (2), we obtain the differential kinematic model:

$$
\boldsymbol{v}_{R}:=\dot{\boldsymbol{p}}_{R}=\frac{\partial \boldsymbol{f}(\boldsymbol{q})}{\partial \boldsymbol{q}} \dot{\boldsymbol{q}}=\boldsymbol{J}(\boldsymbol{q}) \dot{\boldsymbol{q}},
$$

where, $\boldsymbol{v}_{R}=\left[\begin{array}{lll}\boldsymbol{v}_{R 1}^{\top} & \boldsymbol{v}_{R 3}^{\top} & \boldsymbol{v}_{R 5}^{\top}\end{array}\right]^{\top}, \dot{\boldsymbol{q}}=\left[\begin{array}{lll}\boldsymbol{v}_{L}^{\top} & \dot{\boldsymbol{\eta}}_{L}^{\top} & \dot{\boldsymbol{\alpha}}^{\top}\end{array}\right]^{\top} \in$ $\mathbb{R}^{9}$. Equation (3) relates velocities of the platform and cable angles rates to the velocities of the robots. The Jacobian matrix $\boldsymbol{J}(\boldsymbol{q}) \in \mathbb{R}^{9 \times 9}$ is a square invertible matrix in $\mathcal{C}$, except for some singular configurations investigated in [18].

For configurations in which $\boldsymbol{J}(\boldsymbol{q})$ is non-singular, the coordinate $\boldsymbol{p}_{R}$ and its time-derivative $\boldsymbol{v}_{R}$ represent an alternative way to describe the platform configuration $\boldsymbol{q}$ and its velocity $\dot{\boldsymbol{q}}$, respectively, via the inversion of (2) and (3).

In this work, we focus on quasi-static operations for which high-order dynamic effects can be considered negligible. Under this assumption, the kinematic model given by (2) and (3) is enough to describe the system. Nevertheless, possible errors due to this assumption shall be considered in the control design and its stability proof.

\section{ROBUST CONTROL OF THE FLY-CRANE}

We assume that a motion planner, like the one presented in [9], provides at each time $t$ a desired configuration $\boldsymbol{q}^{d}(t)$ that is far from singularities and ensures taut cables.

The proposed control scheme of the Fly-Crane is shown in Fig. 3. From left to right, the desired trajectory quantities $\boldsymbol{q}^{d}, \dot{\boldsymbol{q}}^{d}$ and the measured configuration $\boldsymbol{q}$ are used by the outer configuration controller to generate the reference aerial-vehicle velocities $\boldsymbol{v}_{R}^{\star}$. Using $\boldsymbol{v}_{R}^{\star}$ and the measured velocities $\boldsymbol{v}_{R}$, the velocity controllers of the aerial vehicles compute the commanded accelerations for each vehicle $\boldsymbol{u}=$ $\left[\begin{array}{lll}\boldsymbol{u}_{1}^{\top} & \boldsymbol{u}_{3}^{\top} & \boldsymbol{u}_{5}^{\top}\end{array}\right]^{\top}$. Such commanded accelerations are provided to the low level actuation units of the aerial vehicles, which are in control of the orientation of the vehicles and of the

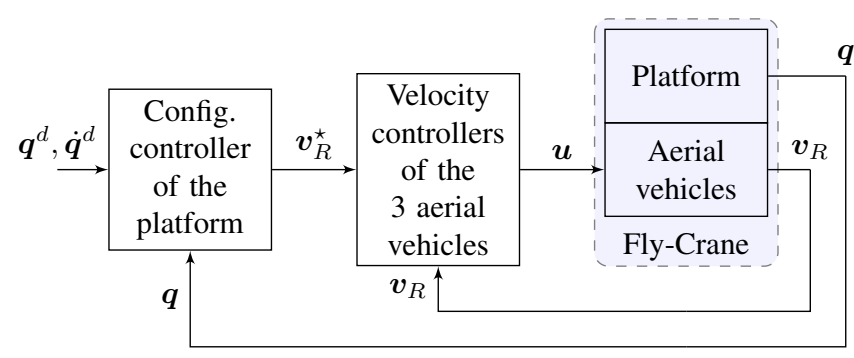

Fig. 3: The control architecture of the Fly-Crane where the configuration controller of the platform and the velocity controllers of the three aerial vehicles are highlighted.

rotor speeds. In the following we detail the equations used in each controller. To face possible errors of the low-level actuation units, an uncertain model for the aerial vehicles is considered.

We denote with $\hat{\boldsymbol{J}}(\boldsymbol{q}) \in \mathbb{R}^{9 \times 9}$ the nominal Jacobian of the system, such that

$$
\hat{\boldsymbol{J}}(\boldsymbol{q})=\boldsymbol{J}(\boldsymbol{q})+\Delta \boldsymbol{J}(\boldsymbol{q}),
$$

where $\Delta \boldsymbol{J}(\boldsymbol{q})$ is the error between the nominal (identified) and real (unkown) Jacobian matrices. Given the desired configuration $\boldsymbol{q}^{d}=\left[\boldsymbol{p}_{L}^{d^{\top}} \boldsymbol{\eta}_{L}^{d^{\top}} \boldsymbol{\alpha}^{d^{\top}}\right]^{\top}$, the corresponding

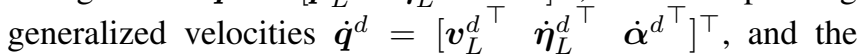
measured configuration $\boldsymbol{q}$, the reference aerial-vehicle velocities are computed:

$$
\boldsymbol{v}_{R}^{\star}=\hat{\boldsymbol{J}}(\boldsymbol{q})\left(\boldsymbol{K}_{\boldsymbol{q}} \boldsymbol{e}_{\boldsymbol{q}}+\dot{\boldsymbol{q}}^{d}\right)
$$

where $^{3} \boldsymbol{K}_{\boldsymbol{q}}=k_{\boldsymbol{q}} \boldsymbol{I}_{9} \in \mathbb{R}_{>0}^{9 \times 9}$ is a positive definite matrix and $\boldsymbol{e}_{\boldsymbol{q}}=\boldsymbol{q}^{d}-\boldsymbol{q}$. The commanded accelerations for the vehicles are computed simply as

$$
\boldsymbol{u}=\boldsymbol{K}_{R}\left(\boldsymbol{v}_{R}^{\star}-\boldsymbol{v}_{R}\right),
$$

where $\boldsymbol{K}_{R}=k_{R} \boldsymbol{I}_{9} \in \mathbb{R}_{>0}^{9 \times 9}$ is a positive definite matrix.

Each aerial vehicle is equipped with a low-level actuation unit that acts on the orientation and intensity of the total force produced by its propellers in order to let $\dot{\boldsymbol{v}}_{R i}$ match the given acceleration input $\boldsymbol{u}_{i}$, for $i=1,2,3$. However, the non perfect knowledge of the platform dynamics and the presence of external disturbances make such matching inexact. The closed-loop dynamics of the vehicle including the actuation unit is then equivalent to an uncertain double integrator, i.e.,

$$
\boldsymbol{v}_{R i}=\dot{\boldsymbol{p}}_{R i}, \quad \dot{\boldsymbol{v}}_{R i}=\boldsymbol{u}_{i}+\boldsymbol{d}_{i}
$$

where the uncontrollable signal $\boldsymbol{d}_{i}$ accounts for all the aforementioned dynamic uncertainties and external disturbances. Such signal $\boldsymbol{d}_{i}$ can be large, small or even close to zero, depending on the type of platform (e.g., under- or fully-actuated), type of controller (e.g., full dynamic model inversion, vehicle model inversion plus disturbance rejection, etc.) and the desired trajectory. Defining the vector $\boldsymbol{d}=$

\footnotetext{
${ }^{3} \boldsymbol{I}_{n} \in \mathbb{R}^{n \times n}$ is the identity matrix of dimension $n$.
} 
$\left[\begin{array}{lll}\boldsymbol{d}_{1}^{\top} & \boldsymbol{d}_{3}^{\top} & \boldsymbol{d}_{5}^{\top}\end{array}\right]^{\top}$ we can write the dynamics of the three aerial vehicles as

$$
\boldsymbol{v}_{R}=\dot{\boldsymbol{p}}_{R}, \quad \dot{\boldsymbol{v}}_{R}=\boldsymbol{u}+\boldsymbol{d} .
$$

Summarizing, the closed loop system dynamics is given by the equations (8), where $\boldsymbol{u}$ is computed using (32) and (5). In such model, the terms $\Delta \boldsymbol{J}(\boldsymbol{q})$ and $\boldsymbol{d}$ account for all the non-idealities arising from modeling errors at the dynamic and kinematic level and the neglected external disturbances. To analyze the system stability under non-ideal conditions, let us write the closed-loop dynamics of the error. Replacing (5) and (32) into (8) and applying the differential kinematics (3) yield

$$
\begin{array}{r}
\boldsymbol{J}(\boldsymbol{q}) \ddot{\boldsymbol{q}}+\dot{\boldsymbol{J}}(\boldsymbol{q}, \dot{\boldsymbol{q}}) \dot{\boldsymbol{q}}=k_{R} \hat{\boldsymbol{J}}(\boldsymbol{q}) k_{\boldsymbol{q}} \boldsymbol{e}_{\boldsymbol{q}}+k_{R} \hat{\boldsymbol{J}}(\boldsymbol{q}) \dot{\boldsymbol{q}}^{d} \\
-k_{R} \boldsymbol{J}(\boldsymbol{q}) \dot{\boldsymbol{q}}+\boldsymbol{d} .
\end{array}
$$

Considering the previous equation with nominal Jacobian as in (4), we can write the error dynamics as

$$
\begin{gathered}
\ddot{\boldsymbol{e}}_{\boldsymbol{q}}=-\left(k_{R} k_{\boldsymbol{q}}+k_{R} k_{\boldsymbol{q}} \boldsymbol{J}^{-1}(\boldsymbol{q}) \Delta \boldsymbol{J}(\boldsymbol{q})\right) \boldsymbol{e}_{\boldsymbol{q}} \\
-\left(k_{R}+\boldsymbol{J}^{-1}(\boldsymbol{q}) \dot{\boldsymbol{J}}(\boldsymbol{q}, \dot{\boldsymbol{q}})\right) \dot{\boldsymbol{e}}_{\boldsymbol{q}}+\boldsymbol{w},
\end{gathered}
$$

where $\boldsymbol{w}$ contains the terms that do not depend on the error and that are considered as disturbances:

$$
\boldsymbol{w}=\ddot{\boldsymbol{q}}^{d}+\boldsymbol{J}^{-1}(\boldsymbol{q})\left(\left(\dot{\boldsymbol{J}}(\boldsymbol{q}, \dot{\boldsymbol{q}})-k_{R} \Delta \boldsymbol{J}(\boldsymbol{q})\right) \dot{\boldsymbol{q}}^{d}-\boldsymbol{d}\right) .
$$

Normally, the control gain $\boldsymbol{K}_{R}$ is tuned independently to (5) to achieve the best tracking of the velocity reference $\boldsymbol{v}_{R}^{\star}$. In this case, we show how to design the configuration control gain $\boldsymbol{K}_{\boldsymbol{q}}=k_{\boldsymbol{q}} \boldsymbol{I}_{9}$, such that to guarantee stability of the closed-loop system with desired $H_{\infty}$ performance, under some sufficient conditions on the uncertainty and disturbance magnitudes.

In the following, for an arbitrary matrix $\boldsymbol{M}, \lambda_{\min }[\boldsymbol{M}]$ and $\lambda_{\max }[\boldsymbol{M}]$ are its minimal and maximal eigenvalues, $\|\boldsymbol{M}\|=$ $\sqrt{\lambda_{\max }\left[\boldsymbol{M}^{\top} \boldsymbol{M}\right]}$ is its induced norm, and $\boldsymbol{M}>0(\boldsymbol{M}<0)$ stands for a positive (negative) definite matrix. $\mathbf{0}_{n} \in \mathbb{R}^{n \times n}$ is the zero matrix of dimension $n \times n$. Finally, with the symbol * we refer to the symmetric term in a matrix.

Theorem 1. Let us consider the control laws (32) and (5) applied to the Fly-Crane under disturbance $\boldsymbol{d}$ and uncertain Jacobian matrix $\hat{\boldsymbol{J}}(\boldsymbol{q}) . k_{R}$ is given while $k_{\boldsymbol{q}}$ has to be designed. Assume that the following bounds hold true for given positive constants $\delta, \iota, \rho \in \mathbb{R}_{>0}$ :

$$
\begin{aligned}
\left\|\Delta \boldsymbol{J}(\boldsymbol{q})^{\top} \Delta \boldsymbol{J}(\boldsymbol{q})\right\| & \leq \delta^{2}, \\
\lambda_{\min }\left[\boldsymbol{J}(\boldsymbol{q})^{\top} \boldsymbol{J}(\boldsymbol{q})\right] & \geq \iota^{2}, \\
\left\|\dot{\boldsymbol{J}}(\boldsymbol{q})^{\top} \dot{\boldsymbol{J}}(\boldsymbol{q})\right\| & \leq \rho^{2} .
\end{aligned}
$$

The designed control gain $k_{\boldsymbol{q}}$ stabilizes the system with $H_{\infty}$ performance defined by

$$
\left\|\boldsymbol{e}_{\boldsymbol{q}}\right\| \leq \gamma\|\boldsymbol{w}\|
$$

with a given constant $\gamma>0$, if there exist scalars $q$ and $q_{k}$ and matrices $\boldsymbol{P}=\boldsymbol{P}^{\top}, \boldsymbol{F}_{1}=\boldsymbol{F}_{1}^{\top}, \boldsymbol{F}_{2}=\boldsymbol{F}_{2}^{\top}, \boldsymbol{G}_{1}=\boldsymbol{G}_{1}^{\top}$, and $\boldsymbol{G}_{2}=\boldsymbol{G}_{2}^{\top}$ of dimension $9 \times 9$ that satisfy the LMI conditions

$$
\left[\begin{array}{ll}
\boldsymbol{P} & q \boldsymbol{I}_{9} \\
* & q \boldsymbol{I}_{9}
\end{array}\right]>0
$$

and

$$
\left[\begin{array}{ccccc}
\boldsymbol{\Xi} & \boldsymbol{E}_{2} q & \boldsymbol{E}_{2} k_{R} q_{k} & \boldsymbol{E}_{1} q & \boldsymbol{E}_{1} k_{R} q_{k} \\
* & -2 \boldsymbol{F}_{1} & \mathbf{0}_{9} & \mathbf{0}_{9} & \mathbf{0}_{9} \\
* & * & -2 \boldsymbol{G}_{1} & \mathbf{0}_{9} & \mathbf{0}_{9} \\
* & * & * & -2 \boldsymbol{F}_{2} & \mathbf{0}_{9} \\
* & * & * & * & -2 \boldsymbol{G}_{2}
\end{array}\right]<0,
$$

with

$$
\boldsymbol{E}_{1}=\left[\begin{array}{lll}
\boldsymbol{I}_{9} & \mathbf{0}_{9} & \mathbf{0}_{9}
\end{array}\right]^{\top}, \quad \boldsymbol{E}_{2}=\left[\begin{array}{lll}
\mathbf{0}_{9} & \boldsymbol{I}_{9} & \mathbf{0}_{9}
\end{array}\right]^{\top},
$$

and $\boldsymbol{\Xi}$ given as

$$
\left[\begin{array}{ccc}
\boldsymbol{\Xi}_{1,1} & \frac{1}{2} \boldsymbol{P}-\frac{1}{2} k_{R} q_{k} \boldsymbol{I}_{9}-\frac{1}{2} k_{R} q \boldsymbol{I}_{9} & q \boldsymbol{I}_{9} \\
* & \frac{\rho^{2}}{2 \iota^{2}}\left(\boldsymbol{F}_{1}+\boldsymbol{F}_{2}\right)-k_{R} q \boldsymbol{I}_{9}+q \boldsymbol{I}_{9} & q \boldsymbol{I}_{9} \\
* & * & -\gamma^{2} \boldsymbol{I}_{9}
\end{array}\right]
$$

with $\boldsymbol{\Xi}_{1,1}=\boldsymbol{I}_{9}+\frac{\delta^{2}}{2 \iota^{2}}\left(\boldsymbol{G}_{1}+\boldsymbol{G}_{2}\right)-k_{R} q \boldsymbol{I}_{9}$. In particular, the designed controller gain $k_{\boldsymbol{q}}$ is then given by $k_{\boldsymbol{q}}=q_{k} / q$.

Proof. Provided in the Appendix.

From a design perspective, given the bounds $\delta, \iota, \rho$ and the velocity controller gain $k_{R}$, we would like to find the $\boldsymbol{K}_{\boldsymbol{q}}$ that provides the best $H_{\infty}$ performance. We can then rewrite Theorem 1 as a constrained minimization problem of $\gamma$ :

$$
\begin{array}{ll}
\underset{\beta}{\operatorname{minimize}} & \gamma \\
\text { subject to } & (16),(17),
\end{array}
$$

where $\beta=\left(\boldsymbol{P}, \boldsymbol{F}_{1}, \boldsymbol{F}_{2}, \boldsymbol{G}_{1}, \boldsymbol{G}_{2}, q, q_{k}, \delta, \iota, \rho, k_{R}, \gamma\right)$. From the solution of (20), and in particular from $q_{k}$ and $q$, the optimal gain $k_{\boldsymbol{q}}$ is computed as $k_{\boldsymbol{q}}=q_{k} / q$.

The main advantage on this method is to find a feasible controller, with the best $H_{\infty}$ gain on view of Theorem 1 , that formally guarantees stability within bounds of uncertainties in the Jacobian matrix $(\delta)$, the proximity to singular configurations $(\iota)$, and its speed $(\rho)$.

Remark. In order to solve (20) a first estimation of the bounds $\delta, \iota$, and $\rho$ is needed. This can be first computed along the desired trajectory knowing the maximum uncertainty on the Jacobian. The latter comes from the imprecision in the design and manufacturing of the platform. Therefore:

$$
\begin{aligned}
& \delta^{2}>\max _{t \in \mathbb{R} \geq 0}\left\|\Delta \boldsymbol{J}\left(\boldsymbol{q}^{d}(t)\right)^{\top} \Delta \boldsymbol{J}\left(\boldsymbol{q}^{d}(t)\right)\right\|, \\
& \iota^{2}<\min _{t \in \mathbb{R} \geq 0} \lambda_{\min }\left[\boldsymbol{J}\left(\boldsymbol{q}^{d}(t)\right)^{\top} \boldsymbol{J}\left(\boldsymbol{q}^{d}(t)\right)\right], \\
& \rho^{2}>\max _{t \in \mathbb{R} \geq 0}\left\|\dot{\boldsymbol{J}}\left(\boldsymbol{q}^{d}(t)\right)^{\top} \dot{\boldsymbol{J}}\left(\boldsymbol{q}^{d}(t)\right)\right\| .
\end{aligned}
$$

Since the bounds strongly depends on the particular trajectory, a motion planner such as the one in [9], could be employed in order to compute the desired trajectory that minimizes the bounds. Notice that also the magnitude of $\boldsymbol{w}$ in (11) depends on the particular motion and especially 
on the system velocity and acceleration. In fact, $\|\boldsymbol{w}\|$ can be bounded as

$$
\|\boldsymbol{w}\| \leq\left\|\ddot{\boldsymbol{q}}^{d}\right\|+\iota\left(\left(\rho+k_{R} \delta\right)\left\|\dot{\boldsymbol{q}}^{d}\right\|+\|\boldsymbol{d}\|\right) .
$$

Therefore, the minimization of $\|\boldsymbol{w}\|$ can also be considered at the planning level. Equation (22) shows that performance is related to desired velocities and accelerations. Since we are more focused on the accuracy rather than on the agility of the maneuvers, we consider that in the domain of interest the motion of the system is done in a quasi-static condition.

Remark. We remark that our controller does not forbid the use a FDM-based method as a low-level controller for the vehicles. This would reduce the velocity tracking error $\boldsymbol{d}_{i}$, improving the overall performance. On the other hand, this would require a precise estimation of the dynamic parameters, which is hard to get as mentioned before.

\section{EXPERIMENTAL RESULTS}

To validate and show the performances of the proposed controller with respect to uncertainties and external disturbances, we designed, implemented and experimentally tested the Fly-Crane in an indoor environment across different conditions.

The Fly-Crane consists of three Quadrotor UAVs connected by six cables to a platform made of carbon fiber bars. The Fly-Crane realization is shown in Fig. 4. Each vehicle weights $1.03[\mathrm{Kg}]$, the platform weights $0.338[\mathrm{Kg}]$, and the length of each cable is $1.2[\mathrm{~m}]$. The aerial vehicles are equipped with a standard flight-controller, four brushless motor controllers regulating the propeller speed in closedloop [19], and an on-board PC that runs the state estimator and the velocity controller ${ }^{4}$. The proposed kinematic controller, implemented in Matlab-Simulink, runs on a desktop PC sending the commanded velocities to the quadrotors through a wifi connection at $100[\mathrm{~Hz}]$. The control loop is then closed based on the estimated state of the vehicles and of the platform. These estimations are computed onboard at $1[\mathrm{kHz}]$ by an UKF that fuses the Motion Capture (MoCap) System measurements (at $120[\mathrm{~Hz}]$ ) with the IMU measurements (at $1[\mathrm{kHz}]$ ).

In the very first phase of each experiment, the platform is lifted from ground, and the system is brought to a non-singular initial configuration. In this phase, the aerial vehicles are independently controlled by a standard position controller. The proposed controller is activated right after.

The trajectory is designed to take the system from its initial configuration, $\boldsymbol{q}^{d}(0)$, to the final desired configuration $\boldsymbol{q}^{d}(T)$ where $\boldsymbol{p}_{L}^{d}=\left[\begin{array}{lll}0.28 & -0.06 & 0.7\end{array}\right]^{\top}[\mathrm{m}], \boldsymbol{\eta}_{L}^{d}=\left[\begin{array}{lll}8 & 13 & 53\end{array}\right]^{\top}[\mathrm{deg}]$, $\boldsymbol{\alpha}^{d}=\left[\begin{array}{lll}32 & 20 & 62\end{array}\right]^{\top}[\mathrm{deg}]$, and $T=20[\mathrm{~s}]$. The gain of the velocity controller is set to $k_{R}=15$ (this value provides the smallest velocity error considering our low-level controller).

Given the desired trajectory and a maximum model uncertainty of $10 \%$ for each parameter, (21) allows computing

\footnotetext{
${ }^{4}$ The full software framework is based on TeleKyb which is open-source and available at https://git.openrobots.org/ projects/telekyb3
}

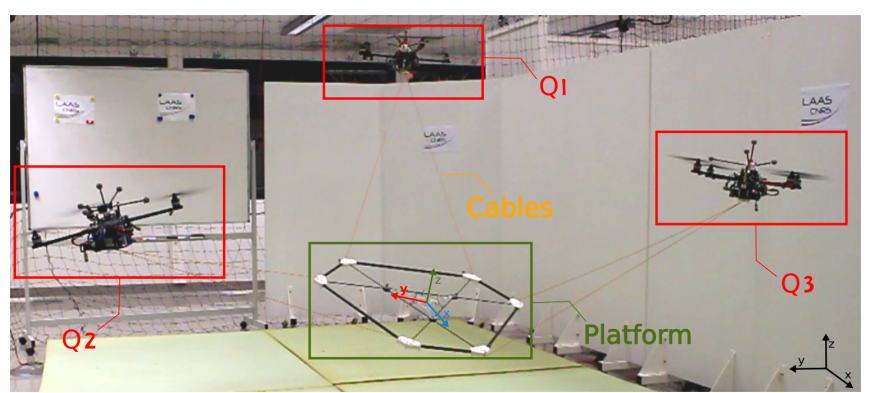

Fig. 4: Experimental screenshot of the Fly-Crane flying while controlling the platform in full pose (position plus orientation).

the bounds needed for the design of $k_{q}$. In particular, the following bounds were considered, $\delta^{2}=0.055, \iota^{2}=0.078$, and $\rho^{2}=0.01$. The gain $k_{\boldsymbol{q}}$ can be then designed using Theorem 1. The solution to the minimization problem (20) provides $k_{\boldsymbol{q}}=0.38$ and $H_{\infty}$ gain $\gamma=1$.

Considering this set-up, we performed three experiments under different conditions:

1) Optimal conditions: the model parameters are the one measured with no additional errors (measurement errors and noise are still present). External disturbances were not applied neither. Due to limited space, we show the relative plots in the attached multimedia material only.

2) Model error: an additional model error in the length of the cables equal to the $10 \%$ of their nominal value is added. The corresponding results are shown in Fig. 5, Fig. 6 and Fig. 7.

3) External disturbance: an artificial force equal to $\left[\begin{array}{lll}1.7 & 1.7 & 1.7\end{array}\right]^{\top}[\mathrm{N}]$ is added to the low level controller of the aerial vehicles. This simulates the effects of external disturbances acting on the vehicles, like wind. The main corresponding results are shown in Fig. 8.

Additional plots are also available in the attached multimedia material together with a video of the conducted experiments.

For experiment 2), in Fig. 5 one can notice that conditions (12), (13) and (14) are always verified. These conditions are fulfilled for experiments 1) and 3) as well. In Fig. 6 and Fig. 8 the norm and the components of the tracking error are shown for experiments 2) and 3), respectively. We can observe that the tracking error does not go exactly to zero, as expected, due to parameter uncertainties, noisy measurements and external disturbances. These non-idealities affects the exactness of the nominal model and the velocity tracking of the aerial vehicles. However, considering the first row of Fig. 6 and Fig. 8 where $\left\|e_{\boldsymbol{q}}\right\|$ and $\gamma\|\boldsymbol{w}\|$ are compared, it is clear that condition (15) is verified and the maximum $H_{\infty}$ performance is guaranteed. We can conclude that, under the fulfillment of conditions (12), (13), and (14) in Theorem 1, the proposed controller and tuning method guarantee the maximum $H_{\infty}$ performance. For experiment 2) only, Fig. 7 shows the desired trajectory with respect to the one performed by the platform. Similar plots corresponding to experiments 1) and 3) are reported in the attached multimedia material.

Although out of the scope of this work, we tested our system for far from quasi-static trajectories. In this 'exper- 


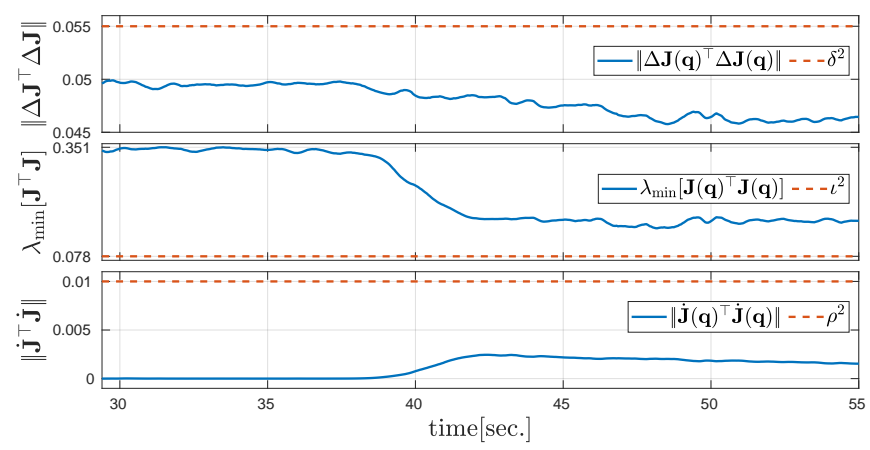

Fig. 5: Verification of conditions (12)-(14) for experiment 2).
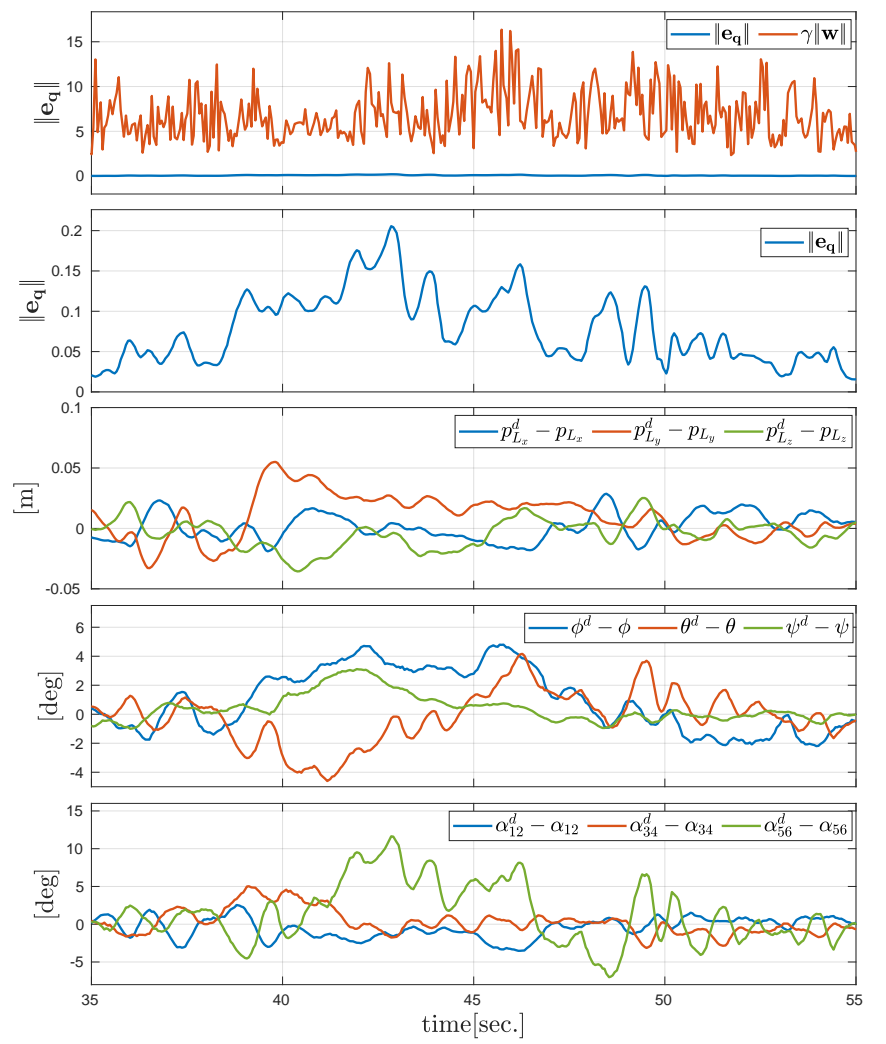

Fig. 6: Tracking error for experiment 2).

iment 4)', we asked the system to accelerate the platform up to $3.2\left[\mathrm{~m} / \mathrm{s}^{2}\right]$ reaching a velocity up to $1.6[\mathrm{~m} / \mathrm{s}]$. As expected, the tracking error increases but the bounds and the $H_{\infty}$ gain of Theorem 1 are still verified. The corresponding plots are reported in the attached multimedia material.

\section{CONCLUSIONS}

A controller capable of dealing with uncertainties in the parameters of the Fly-Crane system has been provided given bounds that can be assessed using the planned trajectory. These bounds can be guaranteed in a real scenario when the Fly-Crane is asked to perform specific trajectories translating and rotating the platform in different directions. The structure of the controller gain $\boldsymbol{K}_{\boldsymbol{q}}$ was limited to a diagonal matrix with equal values to allow design conditions. In the future, we intend to relax this structure and to extend the controller

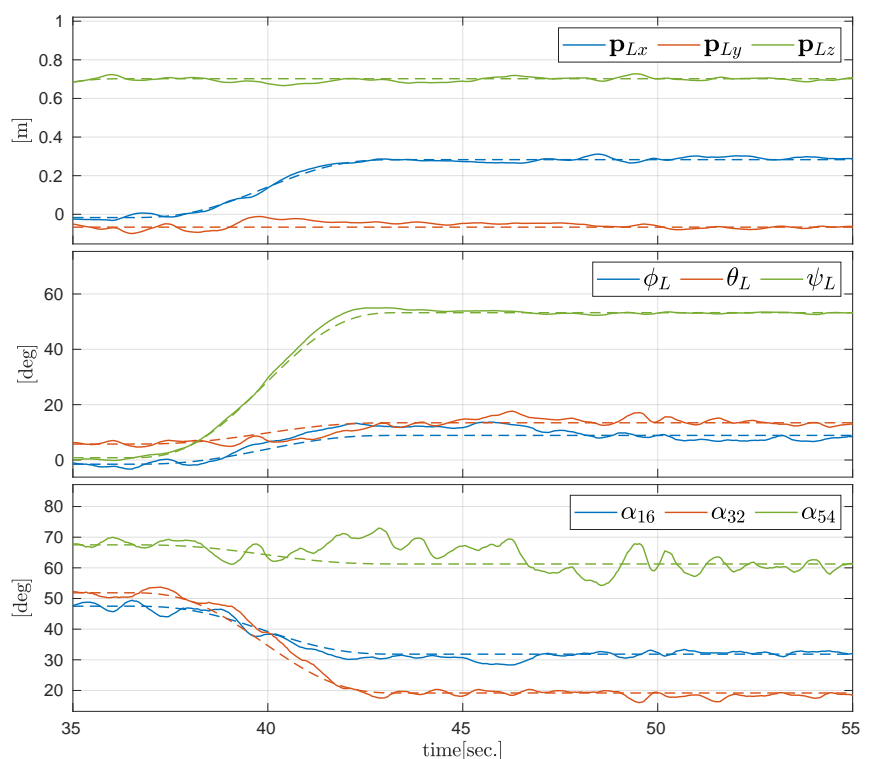

Fig. 7: Tracking of the desired trajectory during system's evolution for experiment 2).

to a second order kinematic approach. The inclusion of a robust dynamic controller is left as future works.

\section{REFERENCES}

[1] I. Palunko, P. Cruz, and R. Fierro, "Agile load transportation: Safe and efficient load manipulation with aerial robots," IEEE Robotics \& Automation Magazine, vol. 19, no. 3, pp. 69-79, 2012.

[2] V. Spurnỳ, T. Báča, M. Saska, R. Pěnička, T. Krajník, J. Thomas, D. Thakur, G. Loianno, and V. Kumar, "Cooperative autonomous search, grasping, and delivering in a treasure hunt scenario by a team of unmanned aerial vehicles," Journal of Field Robotics, vol. 36, no. 1, pp. 125-148, 2019.

[3] F. A. Goodarzi, D. Lee, and T. Lee, "Geometric control of a quadrotor uav transporting a payload connected via flexible cable," International Journal of Control, Automation and Systems, vol. 13, no. 6, pp. 14861498, Dec 2015.

[4] R. Ritz and R. D'Andrea, "Carrying a flexible payload with multiple flying vehicles," in 2013 IEEE/RSJ Int. Conf. on Intelligent Robots and Systems. IEEE, 2013, pp. 3465-3471.

[5] M. Bernard, K. Kondak, I. Maza, and A. Ollero, "Autonomous transportation and deployment with aerial robots for search and rescue missions," Journal of Field Robotics, vol. 28, no. 6, pp. 914-931, 2011.

[6] D. Prattichizzo and J. C. Trinkle, "Grasping," in Springer Handbook of Robotics, B. Siciliano and O. Khatib, Eds. Springer, 2008, pp. 671-700.

[7] K. Sreenath and V. Kumar, "Dynamics, control and planning for cooperative manipulation of payloads suspended by cables from multiple quadrotor robots," in Robotics: Science and Systems, Berlin, Germany, June 2013.

[8] R. Connelly and S. Guest, "Frameworks, tensegrities and symmetry: Understanding stable structures," 2016.

[9] M. Manubens, D. Devaurs, L. Ros, and J. Cortés, "Motion planning for 6-D manipulation with aerial towed-cable systems," in 2013 Robotics: Science and Systems, Berlin, Germany, May 2013.

[10] J. Geng and J. W. Langelaan, "Implementation and demonstration of coordinated transport of a slung load by a team of rotorcraft," in AIAA Scitech 2019 Forum, 2019, p. 0913.

[11] Y. H. Tan, S. Lai, K. Wang, and B. Chen, "Cooperative control of multiple unmanned aerial systems for heavy duty carrying," Annual Reviews in Control, vol. 46, pp. 44-57, 2018.

[12] K. K. Dhiman, A. Abhishek, and M. Kothari, "Cooperative load control and transportation," in 2018 AIAA Information Systems-AIAA Infotech@Aerospace,2018,p. 0895. 


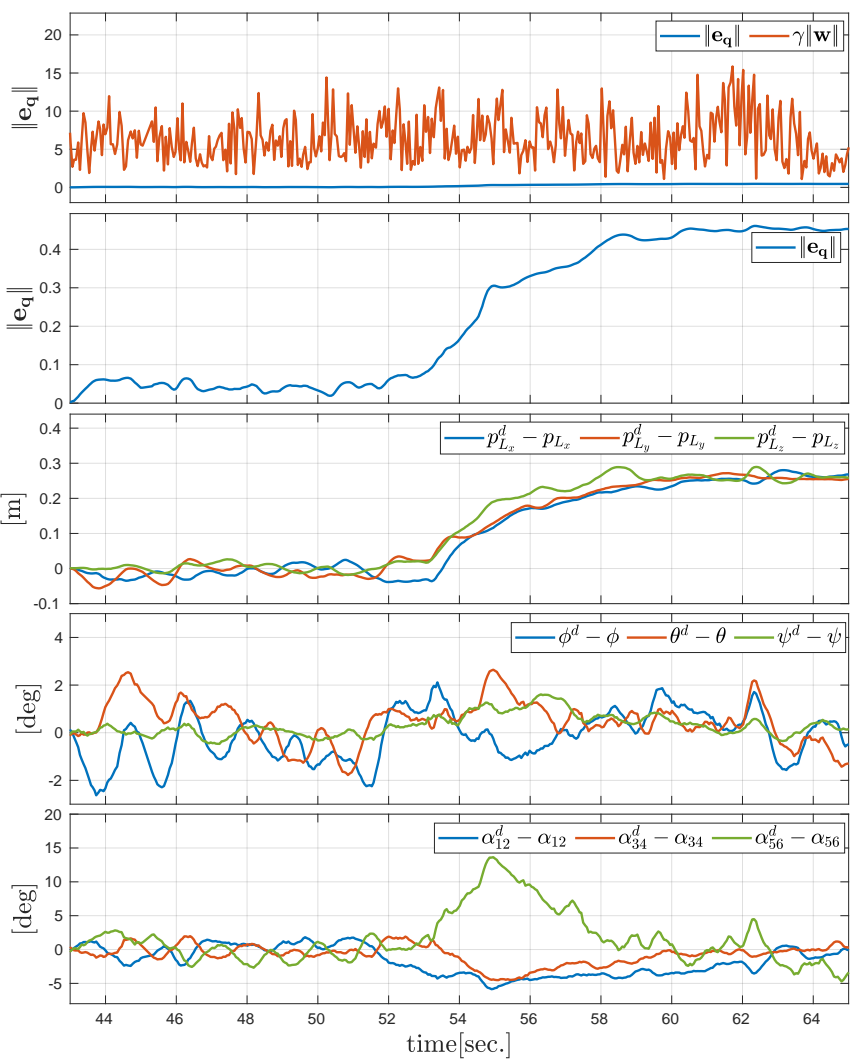

Fig. 8: Tracking error for experiment 3). At time $52[\mathrm{~s}]$ we introduced the virtual disturbance. This cause the increasing of the tracking error in position.

[13] H. G. d. Marina and E. Smeur, "Flexible collaborative transportation by a team of rotorcraft," in 2019 International Conference on Robotics and Automation (ICRA), May 2019, pp. 1074-1080.

[14] M. Tognon, C. Gabellieri, L. Pallottino, and A. Franchi, "Aerial comanipulation with cables: The role of internal force for equilibria, stability, and passivity," IEEE Robotics and Automation Letters, Special Issue on Aerial Manipulation, vol. 3, no. 3, pp. 2577 - 2583, 2018.

[15] T. Lee, "Geometric control of quadrotor uavs transporting a cablesuspended rigid body," IEEE Trans. on Control Systems Technology, vol. 26, no. 1, pp. 255-264, 2017.

[16] C. Masone, H. H. Bülthoff, and P. Stegagno, "Cooperative transportation of a payload using quadrotors: A reconfigurable cable-driven parallel robot," in 2016 IEEE/RSJ Int. Conf. on Intelligent Robots and Systems, Oct 2016, pp. 1623-1630.

[17] S. Ariyibi and O. Tekinalp, "Control of a quadrotor formation carrying a slung load using flexible bars," in AIAA Aviation 2019 Forum, 2019, p. 3270.

[18] D. Six, S. Briot, A. Chriette, and P. Martinet, "The kinematics, dynamics and control of a flying parallel robot with three quadrotors," IEEE Robotics and Automation Letters, vol. 3, no. 1, pp. 559-566, Jan 2018.

[19] A. Franchi and A. Mallet, "Adaptive closed-loop speed control of BLDC motors with applications to multi-rotor aerial vehicles," in 2017 IEEE Int. Conf. on Robotics and Automation, Singapore, May 2017, pp. 5203-5208.

\section{APPENDIX}

\section{A. Proof of Theorem 1}

Consider the Lyapunov candidate function $V\left(\boldsymbol{e}_{\boldsymbol{q}}, \dot{\boldsymbol{e}}_{\boldsymbol{q}}\right)=$ $V_{1}+V_{2}+V_{3}$, with:

$$
V_{1}=\frac{1}{2} \boldsymbol{e}_{\boldsymbol{q}}^{\top} \boldsymbol{P} \boldsymbol{e}_{\boldsymbol{q}}, \quad V_{2}=\frac{1}{2} \dot{\boldsymbol{e}}_{\boldsymbol{q}}^{\top} q \dot{\boldsymbol{e}}_{\boldsymbol{q}}, V_{3}=\boldsymbol{e}_{\boldsymbol{q}}^{\top} q \dot{\boldsymbol{e}}_{\boldsymbol{q}} .
$$

To have $V$ as a Lyapunov function we need $V>0, \forall \boldsymbol{e}_{\boldsymbol{q}} \neq$ $\mathbf{0}, \dot{e}_{\boldsymbol{q}} \neq \mathbf{0}$. Re-writing $V$, this is verified if

$$
V=\frac{1}{2}\left[\begin{array}{c}
\boldsymbol{e}_{\boldsymbol{q}} \\
\dot{\boldsymbol{e}}_{\boldsymbol{q}}
\end{array}\right]^{\top}\left[\begin{array}{cc}
\boldsymbol{P} & q \boldsymbol{I}_{9} \\
* & q \boldsymbol{I}_{9}
\end{array}\right]\left[\begin{array}{c}
\boldsymbol{e}_{\boldsymbol{q}} \\
\dot{\boldsymbol{e}}_{\boldsymbol{q}}
\end{array}\right]>0
$$

which is satisfied only if LMI (16) holds. Next, we consider the time-derivative of $V$. For $V_{1}, \dot{V}_{1}=e_{\boldsymbol{q}}^{\top} \boldsymbol{P} \dot{\boldsymbol{e}}_{\boldsymbol{q}}$. Concerning $V_{2}$, after replacing (10) we obtain

$$
\begin{aligned}
\dot{V}_{Q} \leq & -\dot{\boldsymbol{e}}_{\boldsymbol{q}}^{\top} q k_{R} k_{\boldsymbol{q}} \boldsymbol{J}^{-1}(\boldsymbol{q}) \Delta \boldsymbol{J}(\boldsymbol{q}) \boldsymbol{e}_{\boldsymbol{q}}-\dot{\boldsymbol{e}}_{\boldsymbol{q}}^{\top} q k_{R} k_{\boldsymbol{q}} \boldsymbol{e}_{\boldsymbol{q}} \\
& -\dot{\boldsymbol{e}}_{\boldsymbol{q}}^{\top} q k_{R} \dot{\boldsymbol{e}}_{\boldsymbol{q}}-\dot{\boldsymbol{e}}_{\boldsymbol{q}}^{\top} q \boldsymbol{J}^{-1}(\boldsymbol{q}) \dot{\boldsymbol{J}}(\boldsymbol{q}) \dot{\boldsymbol{e}}_{\boldsymbol{q}}+\dot{\boldsymbol{e}}_{\boldsymbol{q}}^{\top} q \boldsymbol{w} .
\end{aligned}
$$

Given the relation $-2 \boldsymbol{a}^{\top} \boldsymbol{b} \leq \boldsymbol{a}^{\top} \boldsymbol{X}^{-1} \boldsymbol{a}+\boldsymbol{b}^{\top} \boldsymbol{X} \boldsymbol{b}, \boldsymbol{X}>0$, choosing $\boldsymbol{X}$ as $\boldsymbol{F}_{1}>0$, we can use (13) and (14) to bound:

$$
\begin{aligned}
- & \dot{\boldsymbol{e}}_{\boldsymbol{q}}^{\top} q \boldsymbol{J}^{-1}(\boldsymbol{q}) \dot{\boldsymbol{J}}(\boldsymbol{q}) \dot{\boldsymbol{e}}_{\boldsymbol{q}} \leq \frac{1}{2} \dot{\boldsymbol{e}}_{\boldsymbol{q}}^{\top} q \boldsymbol{F}_{1}^{-1} q \dot{\boldsymbol{e}}_{\boldsymbol{q}} \\
+ & \frac{1}{2} \dot{\boldsymbol{e}}_{\boldsymbol{q}}^{\top}\left(\boldsymbol{J}^{-1}(\boldsymbol{q}) \dot{\boldsymbol{J}}(\boldsymbol{q})\right)^{\top} \boldsymbol{F}_{1} \boldsymbol{J}^{-1}(\boldsymbol{q}) \dot{\boldsymbol{J}}(\boldsymbol{q}) \dot{\boldsymbol{e}}_{\boldsymbol{q}} \\
& \leq \frac{1}{2} \dot{\boldsymbol{e}}_{\boldsymbol{q}}^{\top} q \boldsymbol{F}_{1}^{-1} q \dot{\boldsymbol{e}}_{\boldsymbol{q}}+\frac{\rho^{2}}{2 \iota^{2}} \dot{\boldsymbol{e}}_{\boldsymbol{q}}^{\top} \boldsymbol{F}_{1} \dot{\boldsymbol{e}}_{\boldsymbol{q}} .
\end{aligned}
$$

In the same way, choose $\boldsymbol{X}$ as $\boldsymbol{G}_{1}$, with (12), to bound:

$$
\begin{aligned}
& -\dot{\boldsymbol{e}}_{\boldsymbol{q}}^{\top} q k_{R} k_{\boldsymbol{q}} \boldsymbol{J}^{-1}(\boldsymbol{q}) \Delta \boldsymbol{J}(\boldsymbol{q}) \boldsymbol{e}_{\boldsymbol{q}} \\
& \quad \leq \frac{1}{2} \dot{\boldsymbol{e}}_{\boldsymbol{q}}^{\top}\left(q k_{R} k_{\boldsymbol{q}}\right) \boldsymbol{G}_{1}^{-1}\left(q k_{R} k_{\boldsymbol{q}}\right)^{\top} \dot{\boldsymbol{e}}_{\boldsymbol{q}}+\frac{\delta^{2}}{2 \iota^{2}} \boldsymbol{e}_{\boldsymbol{q}}^{\top} \boldsymbol{G}_{1} \boldsymbol{e}_{\boldsymbol{q}} .
\end{aligned}
$$

Finally, we have that the derivative of $V_{2}$ is bounded by

$$
\begin{aligned}
\dot{V}_{Q} \leq & \frac{1}{2} \dot{\boldsymbol{e}}_{\boldsymbol{q}}^{\top}\left(q k_{R} k_{\boldsymbol{q}}\right) \boldsymbol{G}_{1}^{-1}\left(q k_{R} k_{\boldsymbol{q}}\right)^{\top} \dot{\boldsymbol{e}}_{\boldsymbol{q}}+\frac{\delta^{2}}{2 \iota^{2}} \boldsymbol{e}_{\boldsymbol{q}}^{\top} \boldsymbol{G}_{1} \boldsymbol{e}_{\boldsymbol{q}} \\
& -\dot{\boldsymbol{e}}_{\boldsymbol{q}}^{\top} q k_{R} k_{\boldsymbol{q}} \boldsymbol{e}_{\boldsymbol{q}}-\dot{\boldsymbol{e}}_{\boldsymbol{q}}^{\top} q k_{R} \dot{\boldsymbol{e}}_{\boldsymbol{q}} \\
& +\frac{1}{2} \dot{\boldsymbol{e}}_{\boldsymbol{q}}^{\top} q \boldsymbol{F}_{1}^{-1} q \dot{\boldsymbol{e}}_{\boldsymbol{q}}+\frac{\rho^{2}}{2 \iota^{2}} \dot{\boldsymbol{e}}_{\boldsymbol{q}}^{\top} \boldsymbol{F}_{1} \dot{\boldsymbol{e}}_{\boldsymbol{q}}+\dot{\boldsymbol{e}}_{\boldsymbol{q}}^{\top} q \boldsymbol{w} .
\end{aligned}
$$

Taking the time-derivative of term $V_{3}$ and proceeding analogously, for some $\boldsymbol{F}_{2}>0$ and $\boldsymbol{G}_{2}>0$, we can obtain

$$
\begin{aligned}
\dot{V}_{R} \leq & \frac{1}{2} \boldsymbol{e}_{\boldsymbol{q}}^{\top}\left(q k_{R} k_{\boldsymbol{q}}\right) \boldsymbol{G}_{2}^{-1}\left(q k_{R} k_{\boldsymbol{q}}\right)^{\top} \boldsymbol{e}_{\boldsymbol{q}}+\frac{\delta^{2}}{2 \iota^{2}} \boldsymbol{e}_{\boldsymbol{q}}^{\top} \boldsymbol{G}_{2} \boldsymbol{e}_{\boldsymbol{q}} \\
& -\boldsymbol{e}_{\boldsymbol{q}}^{\top} q k_{R} k_{\boldsymbol{q}} \boldsymbol{e}_{\boldsymbol{q}}-\boldsymbol{e}_{\boldsymbol{q}}^{\top} q k_{R} \dot{\boldsymbol{e}}_{\boldsymbol{q}}+\dot{\boldsymbol{e}}_{\boldsymbol{q}}^{\top} q \dot{\boldsymbol{e}}_{\boldsymbol{q}} \\
& +\frac{1}{2} \boldsymbol{e}_{\boldsymbol{q}}^{\top} q \boldsymbol{F}_{2}^{-1} q \boldsymbol{e}_{\boldsymbol{q}}+\frac{\rho^{2}}{2 \iota^{2}} \dot{\boldsymbol{e}}_{\boldsymbol{q}}^{\top} \boldsymbol{F}_{2} \dot{\boldsymbol{e}}_{\boldsymbol{q}}+\boldsymbol{e}_{\boldsymbol{q}}^{\top} q \boldsymbol{w} .
\end{aligned}
$$

To show that stability with the $H_{\infty}$ performance is satisfied we use the following condition:

$$
\boldsymbol{e}_{\boldsymbol{q}}^{\top} \boldsymbol{e}_{\boldsymbol{q}}-\gamma^{2} \boldsymbol{w}^{\top} \boldsymbol{w}+\dot{V}<0,
$$

given the Lyapunov candidate function $V$ and a scalar $\gamma>0$. Integrating both sides in any time-interval $[0, T]$ and assuming zero initial conditions $\boldsymbol{e}_{\boldsymbol{q}}(0)=\mathbf{0}$ and $\dot{\boldsymbol{e}}_{\boldsymbol{q}}(0)=\mathbf{0}$, we have

$$
\int_{0}^{T}\left(\boldsymbol{e}_{\boldsymbol{q}}^{\top} \boldsymbol{e}_{\boldsymbol{q}}-\gamma^{2} \boldsymbol{w}^{\top} \boldsymbol{w}\right) d t+V\left(\boldsymbol{e}_{\boldsymbol{q}}(T), \dot{e}_{\boldsymbol{q}}(T)\right)<0,
$$

such that, if condition (29) is satisfied then (15) holds and the system is stable with $H_{\infty}$ performance.

To verify condition (29), since $\dot{V}=\dot{V}_{1}+\dot{V}_{2}+\dot{V}_{3}$, we use the upper bounds in (27) and (28) into (29) to write

$$
\boldsymbol{\xi}^{\top} \boldsymbol{A} \boldsymbol{\xi}<0
$$


with $\boldsymbol{\xi}^{\top}=\left[\boldsymbol{e}_{\boldsymbol{q}}^{\top} \dot{\boldsymbol{e}}_{\boldsymbol{q}}^{\top} \boldsymbol{w}^{\top}\right]$ $\boldsymbol{A}=\left[\boldsymbol{\Xi}+\boldsymbol{E}_{2} q \frac{1}{2} \boldsymbol{F}_{1}^{-1}\left(\boldsymbol{E}_{2} q\right)^{\top}+\boldsymbol{E}_{2} q k_{R} k_{\boldsymbol{q}} \frac{1}{2} \boldsymbol{G}_{1}^{-1}\left(\boldsymbol{E}_{2} q k_{R} k_{\boldsymbol{q}}\right)^{\top}\right.$ $\left.+\boldsymbol{E}_{1} q \frac{1}{2} \boldsymbol{F}_{2}^{-1}\left(\boldsymbol{E}_{1} q\right)^{\top}+\boldsymbol{E}_{1} q k_{R} k_{\boldsymbol{q}} \frac{1}{2} \boldsymbol{G}_{2}^{-1}\left(\boldsymbol{E}_{1} q k_{R} k_{\boldsymbol{q}}\right)^{\top}\right]$,

$\boldsymbol{\Xi}$ given in (19), and $\boldsymbol{E}_{1}$ and $\boldsymbol{E}_{2}$ in (18). Thus, for the condition (31) to hold, $\boldsymbol{A}$ has to be imposed negative definite. Applying the Schur's Complement on this term, and writing $q_{k}=q k_{\boldsymbol{q}}$ to allow for control design, yields the LMI condition in (17). If the LMI conditions are satisfied, $k_{\boldsymbol{q}}$ can be obtained by taking $k_{\boldsymbol{q}}=q_{k} / q$. This completes the proof.

\section{B. Additional experimental results}

In Sec. IV we mentioned some experimental results concerning the demonstration of the robustness of our controller in different possible scenarios and tracking different trajectories. Here we provide additional results and details of the presented experimental. Considering the experimental set-up described in Sec. IV, in the following we provide additional results for the four experiments:

1) Optimal conditions: the model parameters are the one measured with no additional errors (non-zero measurement errors and noise are still present). External disturbances were not applied neither.

2) Model error: an additional model error in the length of the cables equal to the $10 \%$ of their nominal value is added.

3) External disturbance: an artificial force equal to $\left[\begin{array}{lll}1.7 & 1.7 & 1.7\end{array}\right]^{\top}[\mathrm{N}]$ is added to the low level controller of the aerial vehicles. This simulates the effects of external disturbances acting on the vehicles, like wind.

4) Track of a far from quasi-static trajectory: we asked the system to accelerate the platform up to $3.2\left[\mathrm{~m} / \mathrm{s}^{2}\right]$ reaching a velocity up to $1.6[\mathrm{~m} / \mathrm{s}]$.

Our intent is to provide, for each experiment, the following results related to:

1) comparison between $\boldsymbol{q}^{d}$ and $\boldsymbol{q}$;

2) tracking errors;

3) desired and actual vehicle velocities, $\boldsymbol{v}_{R}^{\star}$ and $\boldsymbol{v}_{R}$, respectively.

1) Optimal Conditions: The model parameters are the one measured with no additional errors (measurement errors and noise are still present). External disturbances were not applied neither. From Fig. 9 one can observe that the controller is able to steer the system to the desired configuration with a error less than $0.02[\mathrm{~m}]$ in position, $2^{\circ}$ in the attitude and $2^{\circ}$ in the cables angle, $\boldsymbol{\alpha}$.

2) Model error: From Fig. 2 we can observe that the tracking error does not go perfectly to zero due to parameter uncertainties, noisy measurements and external disturbances. These non-idealities affects the exactness of the nominal model and the velocity tracking of the aerial vehicles. However, looking at $\left\|\boldsymbol{e}_{\boldsymbol{q}}\right\|$ and $\gamma\|\boldsymbol{w}\|$, it is clear that condition of Theorem 1 is verified and the maximum $H_{\infty}$ performance is guaranteed.
3) Simulated Constant Disturbance: We tested our system when an an artificial force equal to $\boldsymbol{f}_{\text {ext }}=\left[\begin{array}{lll}1.7 & 1.7 & 1.7\end{array}\right]^{\top}[\mathrm{N}]$ is added to the low level controller of the aerial vehicles:

$$
\boldsymbol{u}_{i}=k_{R}\left(\boldsymbol{v}_{R i}^{\star}-\boldsymbol{v}_{R i}\right)+\frac{\boldsymbol{f}_{\text {ext }}}{m_{R_{i}}}
$$

where $m_{R_{i}}$ is the mass of the $i$-th vehicle.

As a consequence of this external disturbance, one can observe from Fig. 11 that the position error of the platform increases. Nevertheless, the bounds are largely respected and $\left\|\boldsymbol{e}_{\boldsymbol{q}}\right\|$ remains bounded by $\gamma\|\boldsymbol{w}\|$.

4) Fast Trajectory Experiment: With this experiment, we tested the robustness of the proposed method when the assumption of quasi-static trajectory is largely violated, i.e., when the system is asked to follow a dynamic trajectories. In doing so, we aim at understanding the limits of our method. Nevertheless, we remark that our focus is on accurate tasks where slow trajectories are preferred with respect to fast ones. The trajectory is designed to take the system from the starting configuration to a successive configurations of

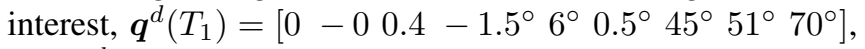

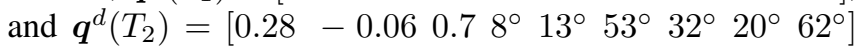
where $T_{1}=42$ and $T_{2}=48$. As the reader can see in Fig. 12 the developed controller is able to quickly follow, once again, the given reference trajectory trying to minimize the error. Even though the $\left\|\boldsymbol{e}_{\boldsymbol{q}}\right\|$ increases as expected during the dynamic part of the trajectory, it still remains bounded by $\gamma\|\boldsymbol{w}\|$ and goes back to smaller values when the trajectory is static. 


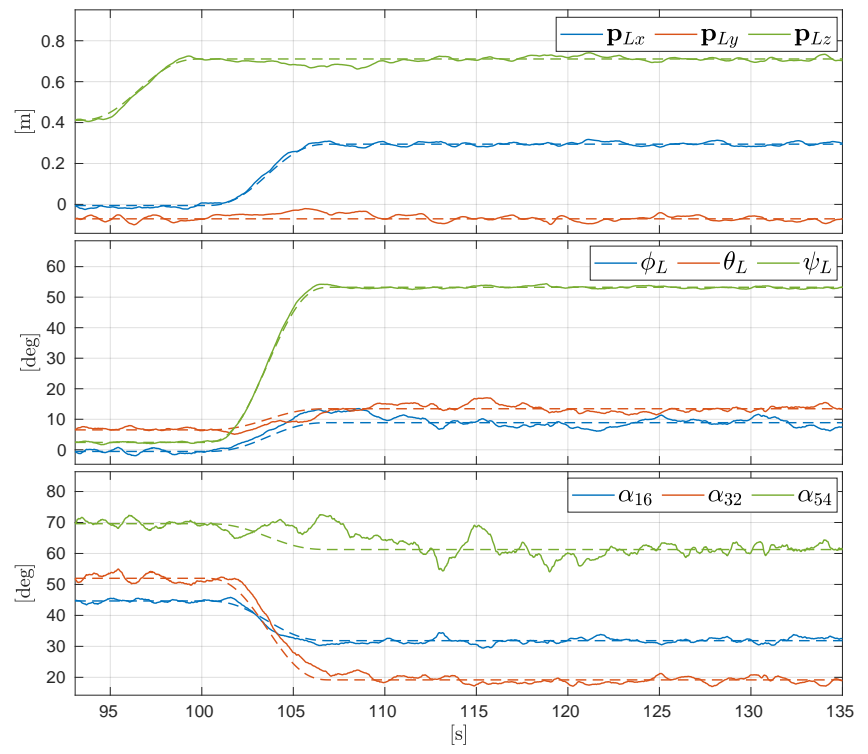

(a) Tracking of the desired trajectory.
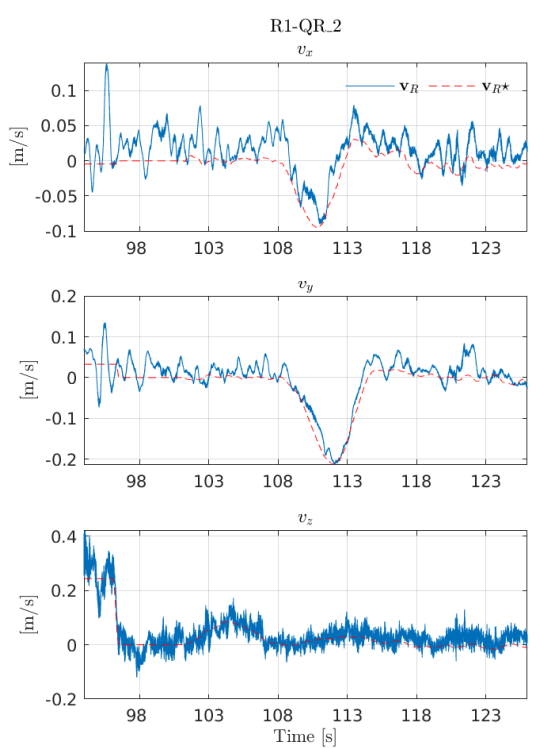

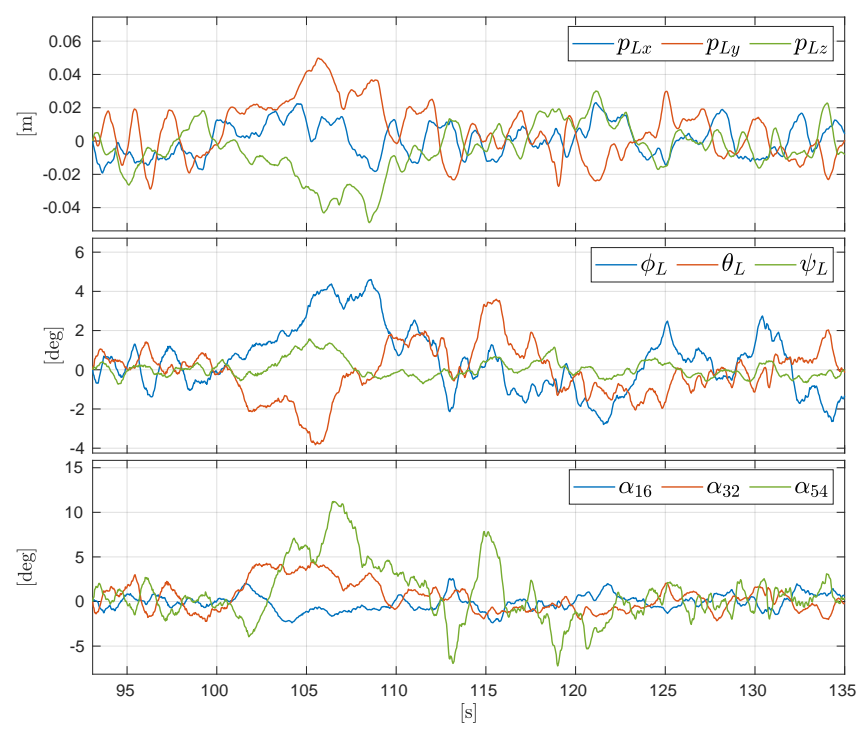

(b) Tracking errors.
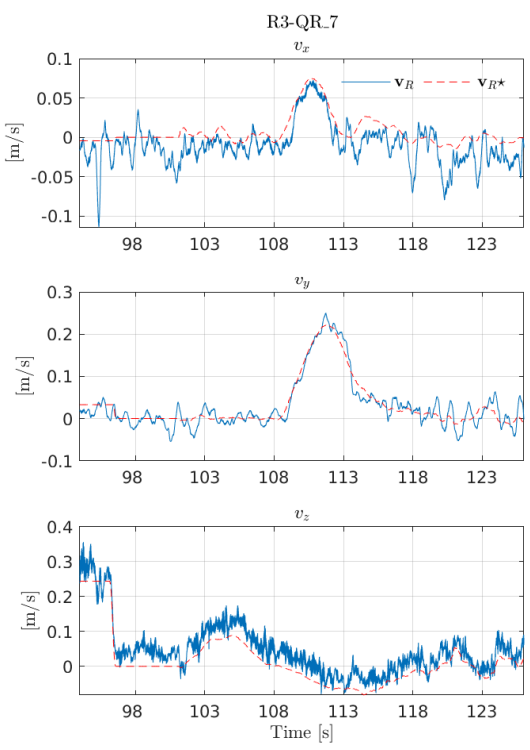

(c) Velocity tracking for the aerial vehicles.

Fig. 9: Plots for experiment 1) 


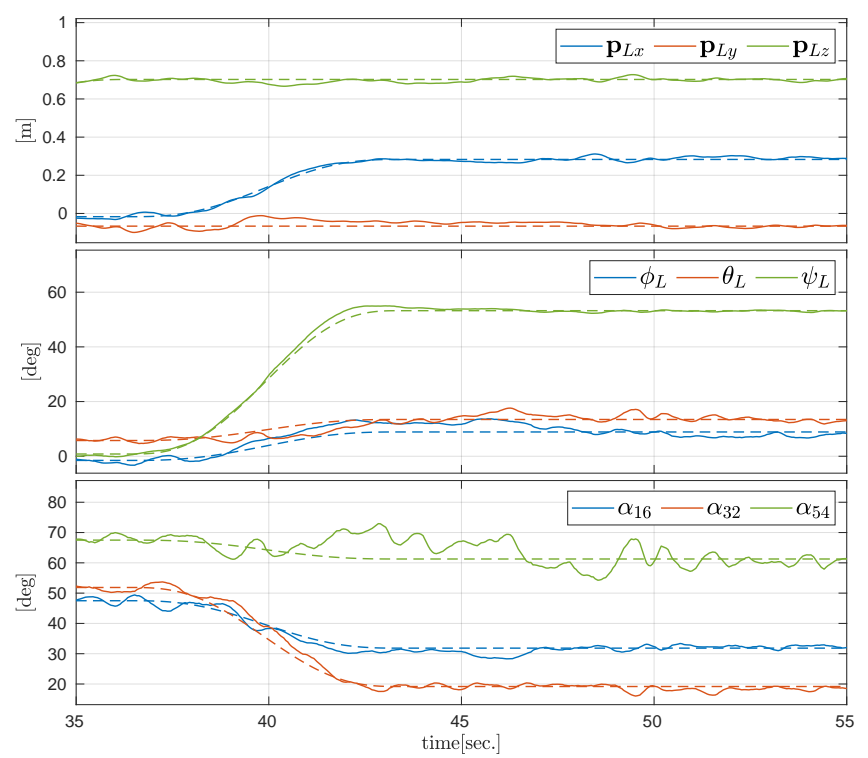

(a) Tracking of the desired trajectory.
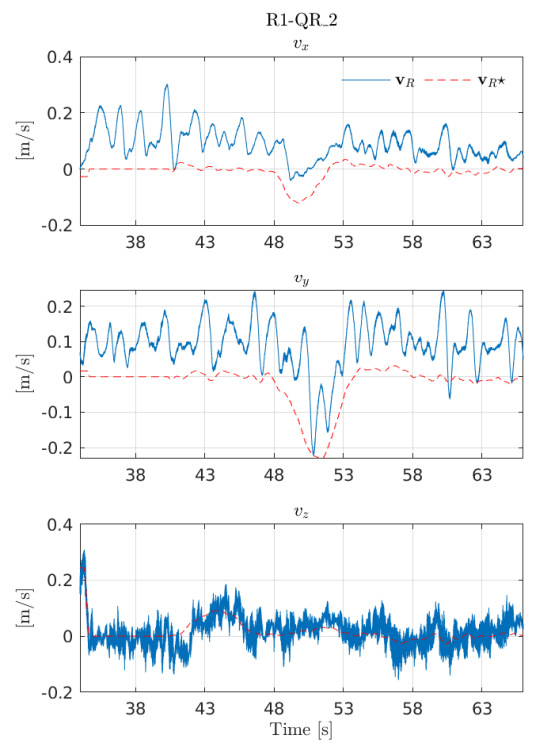
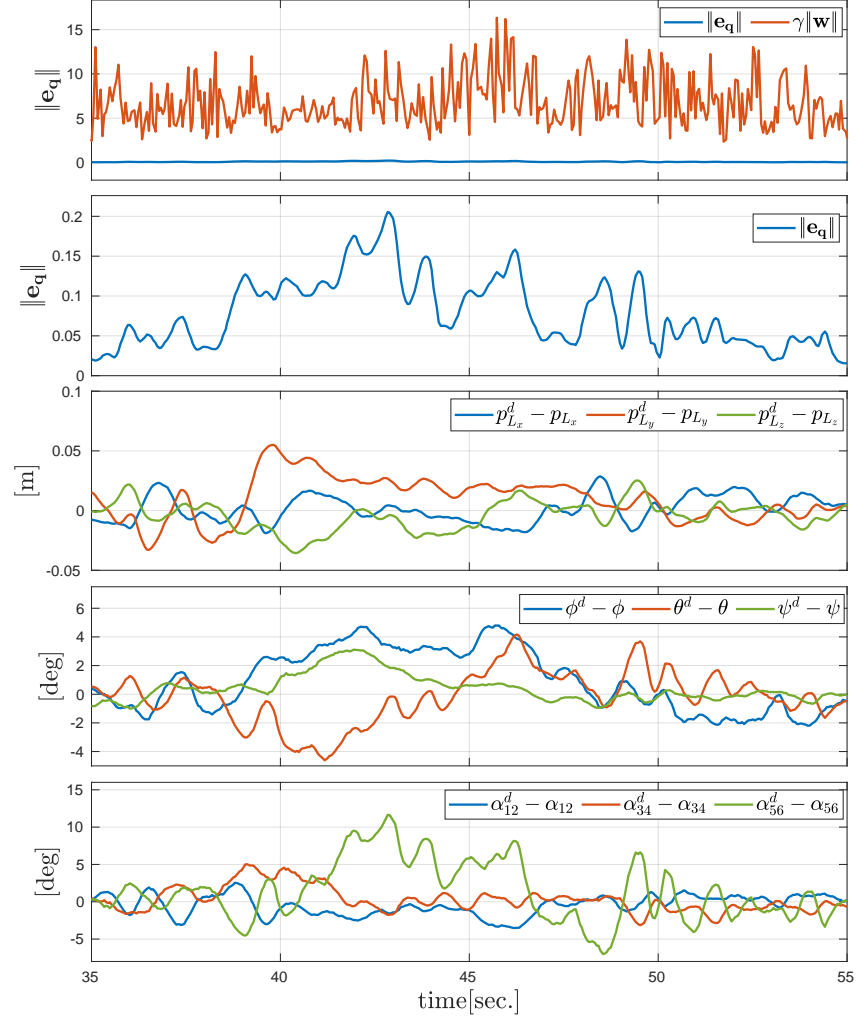

(b) Tracking errors.
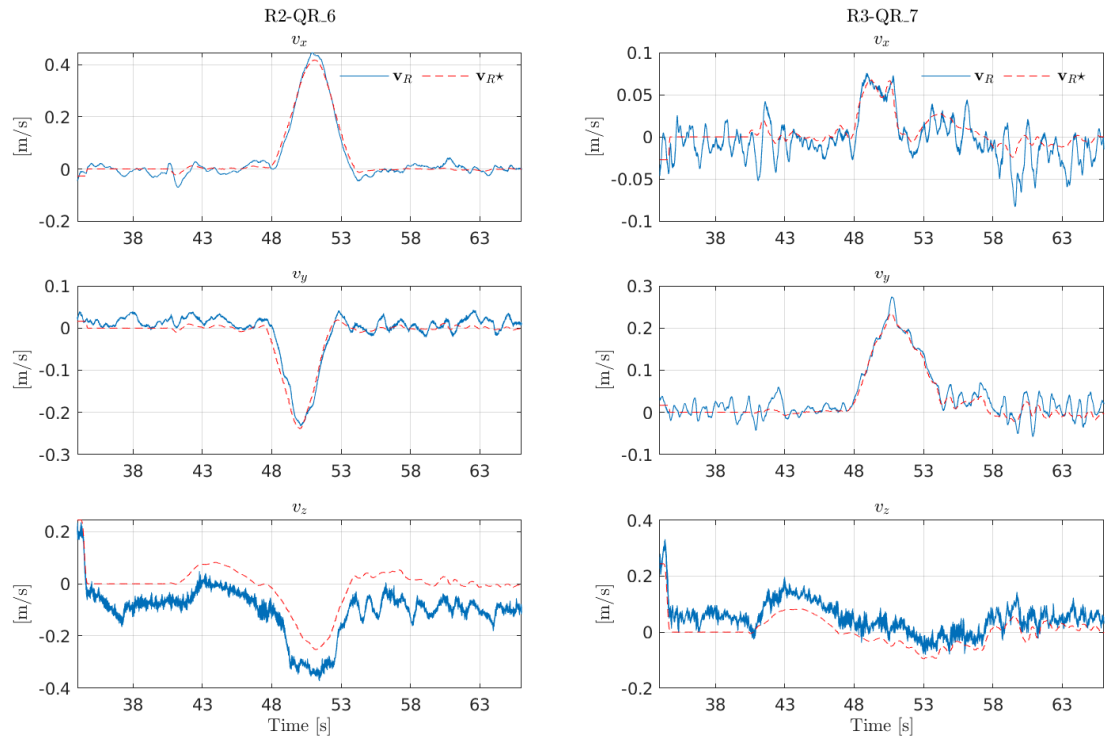

(c) Velocity tracking for the aerial vehicles.

Fig. 10: Plots for experiment 2) 


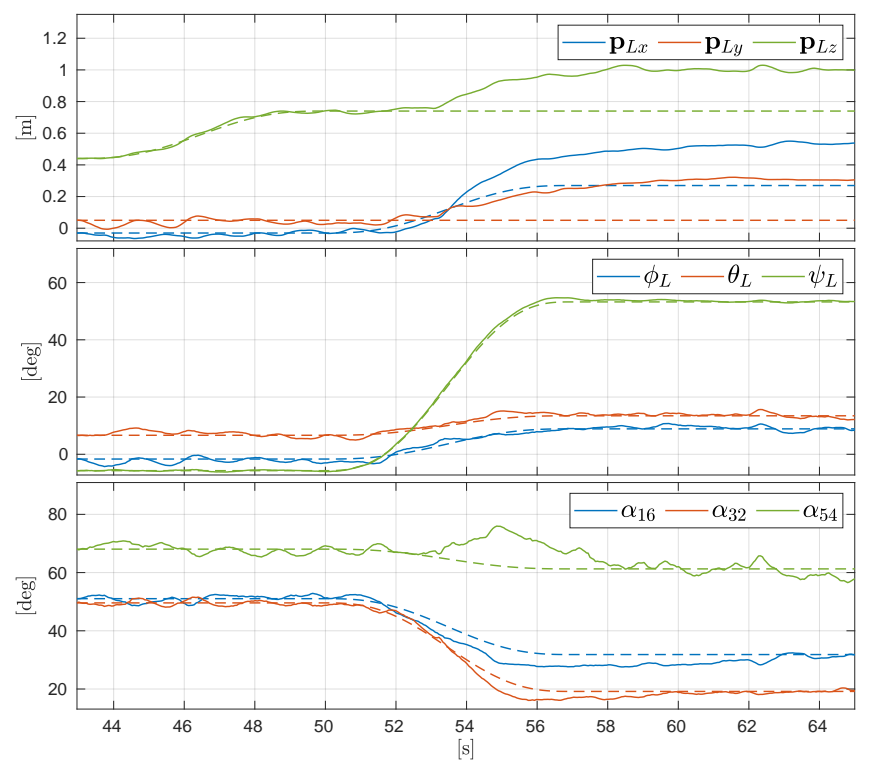

(a) Tracking of the desired trajectory.
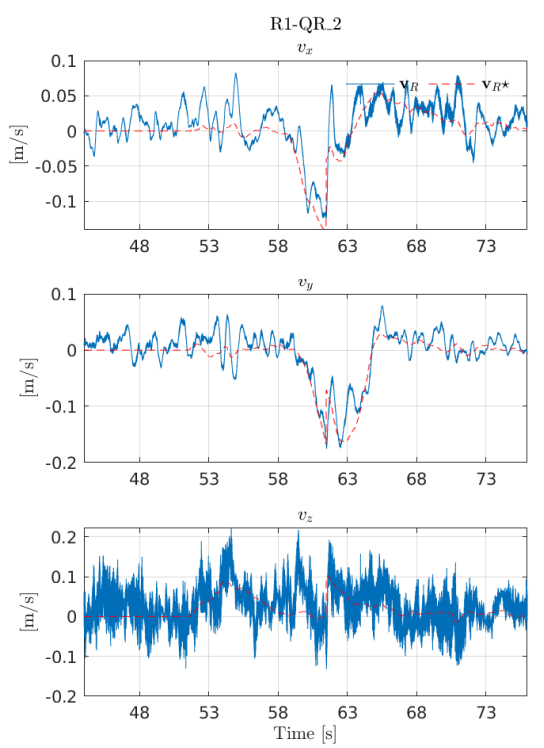
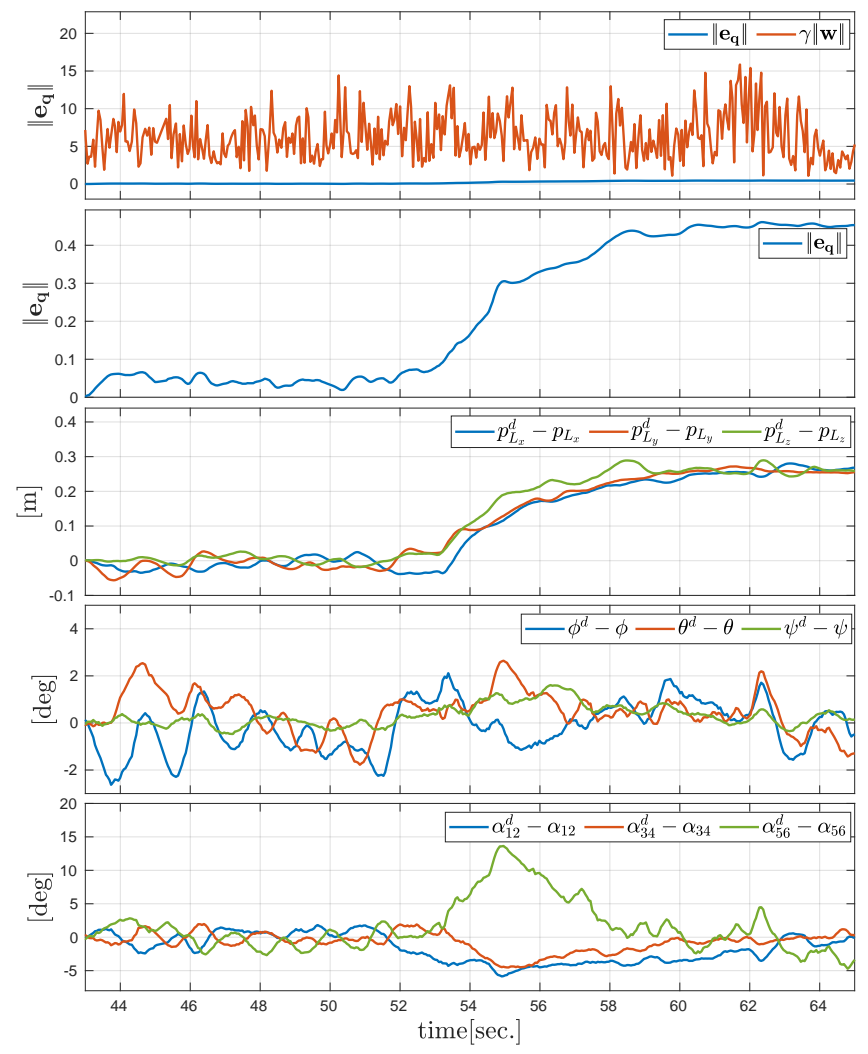

(b) Tracking errors.
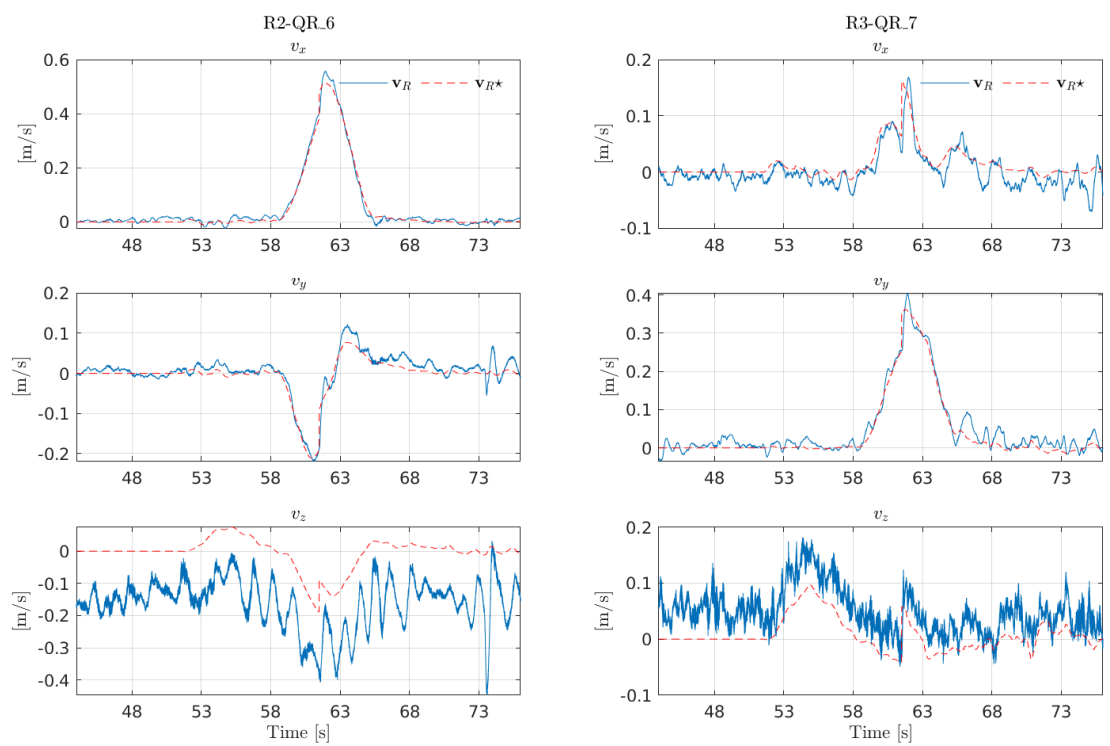

(c) Velocity tracking for the aerial vehicles.

Fig. 11: Plots for experiment 3) 


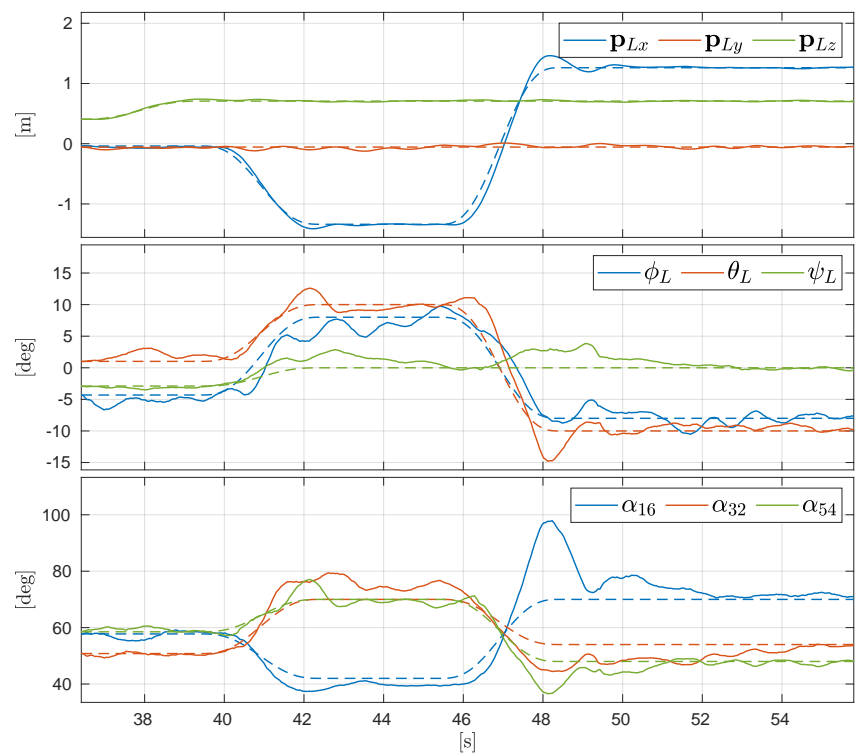

(a) Tracking of the desired trajectory.
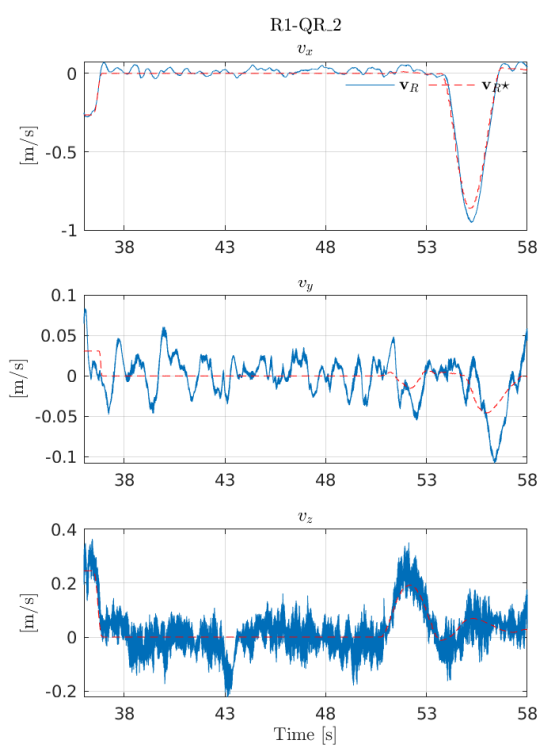
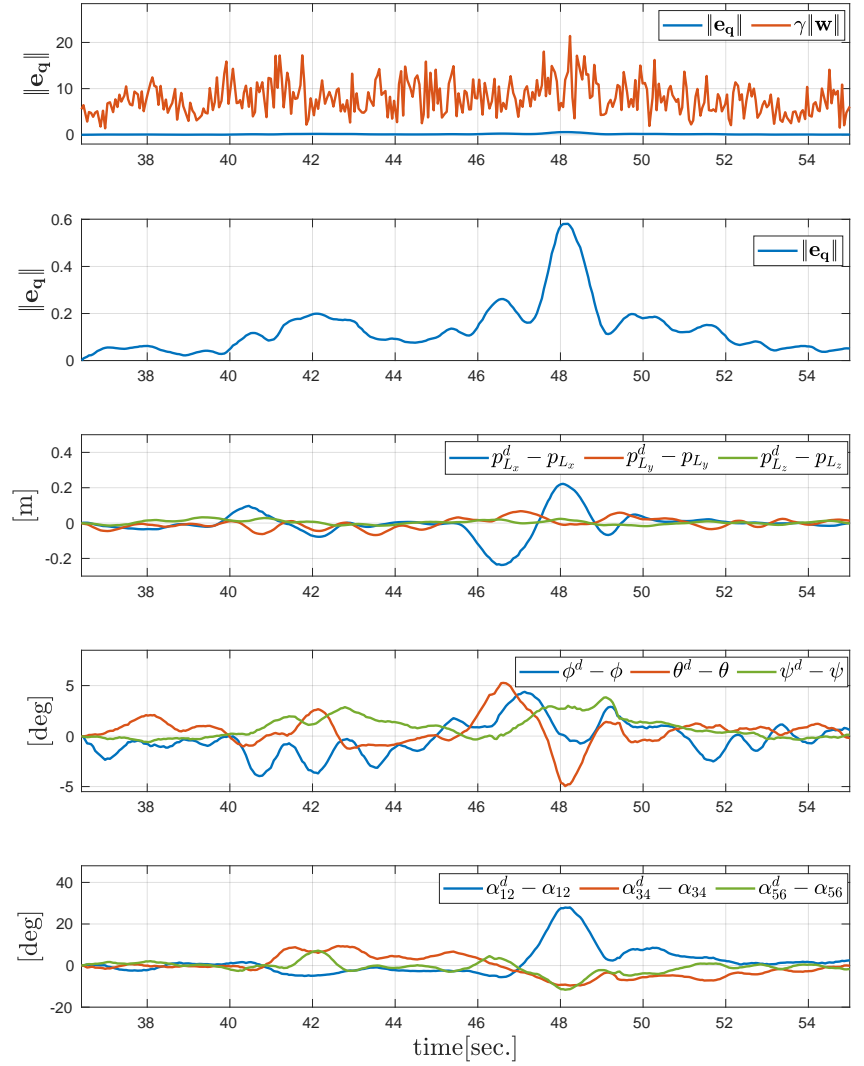

(b) Tracking errors.
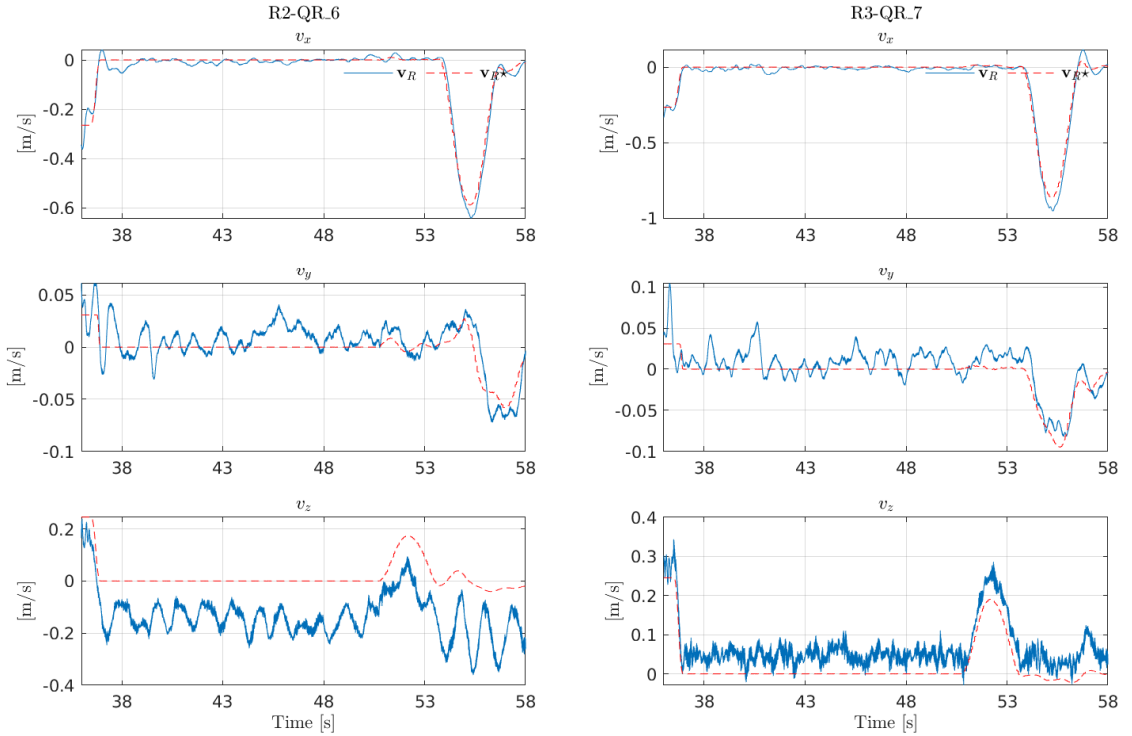

(c) Velocity tracking for the aerial vehicles.

Fig. 12: Plots for experiment 4) 